\title{
DESINGULARIZATION OF IDEALS AND VARIETIES
}

\author{
HERWIG HAUSER
}

University of Innsbruck, Austria

\begin{abstract}
Singular mobiles were introduced by Encinas and Hauser in order to conceptualize the information which is necessary to prove strong resolution of singularities in characteristic zero. It turns out that after Hironaka's Annals paper from 1964 essentially all proofs rely - either implicitly or explicitly - on the data collected in a mobile, often with only small technical variations. The present text explains why mobiles are the appropriate resolution datum and how they are used to build up the induction argument of the proof.
\end{abstract}

Keywords: resolution, blowups, singularities.

\section{CLASS 1: Examples etc.}

The Cylinder $X_{1}: x^{2}+y^{2}=1$ in $\mathbb{A}^{3}$ contracts under $(x, y, z) \rightarrow(x z, y z, z)$ to the Cone $X_{2}: x^{2}+y^{2}=z^{2}$. The linear change $(x, y, z) \rightarrow(x, 2 y, z+y)$ transforms this equation into $X_{2}: x^{2}+(y-z) z=0$. This Cone contracts under $(x, y, z) \rightarrow(x y, y, y z)$ to the Calypso $X_{3}: x^{2}+y^{2} z=z^{2}$. From there, we get via $(x, y, z) \rightarrow(x z, y, z)$ the Calyx of equation $X_{4}: x^{2}+y^{2} z^{3}=z^{4}$. In this way, the Calyx is represented as the image of a smooth scheme under a rational map. We have parametrized a singular surface by a regular one (see fig. 1-4).
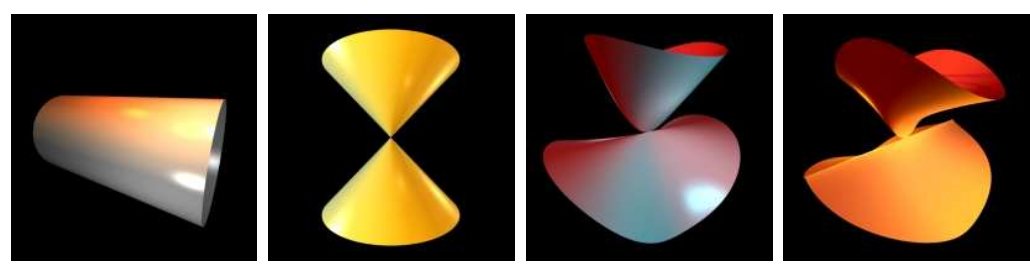

Figures 1-4: Resolution of Calyx by three successive blowups. 
Start now with Calyx. We propose in this course to describe methods which allow to reconstruct from its equation the above or some other paramatrization.

Setting: $X$ reduced singular scheme of finite type over a field $K$, mostly affine, $X=\operatorname{Spec} A$, with $A$ a nilpotent-free finitely generated $K$-algebra. Choose a presentation $K\left[x_{1}, \ldots, x_{n}\right] \rightarrow A=K\left[x_{1}, \ldots, x_{n}\right] / I$ for some ideal $I$ of $K[x]=K\left[x_{1}, \ldots, x_{n}\right]$. This corresponds to an embedding $X \subset \mathbb{A}^{n}=$ $\mathbb{A}_{K}^{n}$ with $X=V(I)$. We may also choose generators $I=\left(g_{1}, \ldots, g_{k}\right)$. The singular locus $\operatorname{Sing} X$ of $X$ is a closed reduced subscheme of $X$.

Example: The Spitz of equation $\left(z^{3}-x^{2}-y^{2}\right)^{3}=x^{2} y^{2} z^{3}$ in $\mathbb{A}^{3}$ (fig. 7). The singular locus consists of two cusps (one in $x z$-plane, one in $y z$-plane) with the same tangent at 0 . Isomorphic to the cartesian product of plain cusp with itself.

Resolution of singularities: Surjective morphism $\tilde{X} \rightarrow X$ with $\tilde{X}$ regular. Also: Desingularization, parametrization, projection, shadow.

Embedded resolution: Given $X$ in a regular $W$, a proper birational morphism $\Pi: \tilde{W} \rightarrow W$ and a regular $\tilde{X} \subset \tilde{W}$ which maps under $\Pi$ onto $X$ and is transversal to exceptional divisor $E=\pi^{-1} Z$, where $Z \subset W$ is the locus above which $\pi$ is not an isomorphism (usually: $Z=\operatorname{Sing} X$ ).

Strong resolution of $X \subset W:$ Embedded resolution $\pi: \tilde{X} \rightarrow X$ induced by $\Pi: \tilde{W} \rightarrow W$ such that:

- $\pi$ isomorphism outside $\operatorname{Sing} X$ (economy);

- $\pi$ independent of embedding $X \subset W$ (excision);

- $\pi$ commutes with smooth morphisms (equivariance), in particular with open immersions, localization, completion, with taking cartesian product with regular scheme, field extensions, group actions on $X$ lift to action on $\tilde{X}$;

- $\pi$ is composition of blowups in regular centers (explicitness);

- centers of blowup are the top locus of a local upper semicontinuous invariant (effectiveness).

Exercises: (1) Prove that the maps given at the very beginning yield indeed a resolution of the Calyx. Show that all properties of an embedded resolution are fulfilled. Determine the centers of blowup as well as all exceptional components. 
(2) Find for the Kolibri of equation $x^{2}=y^{2} z^{2}+z^{3}$ a resolution (fig. 5). Determine first the geometry and the singular locus. Try as first centers both the origin and the singular locus.

(3) Show that the map $\mathbb{A}^{2} \rightarrow \mathbb{A}^{3}$ given by $(s, t) \rightarrow\left(s t, s, t^{2}\right)$ parametrizes the Whitney-umbrella $X: x^{2}=y^{2} z$ (fig. 6). Is it a resolution? Check if all required properties hold.

(4) Show that the blowup of the Whitney-umbrella with center the origin yields a surface which has one cone-like isolated singularity and at another point the singularity of the Whitney-umbrella (fig. 6'). Conclude from this that the singularities need not improve if the centers are too small.

(5) Determine all finite symmetries of the Spitz (fig. 7). Then show that it is isomorphic to the cartesian product of the cusp $x^{2}=y^{3}$ in the plane $\mathbb{A}^{2}$ with itself. Find other embeddings of this product into $\mathbb{A}^{3}$.
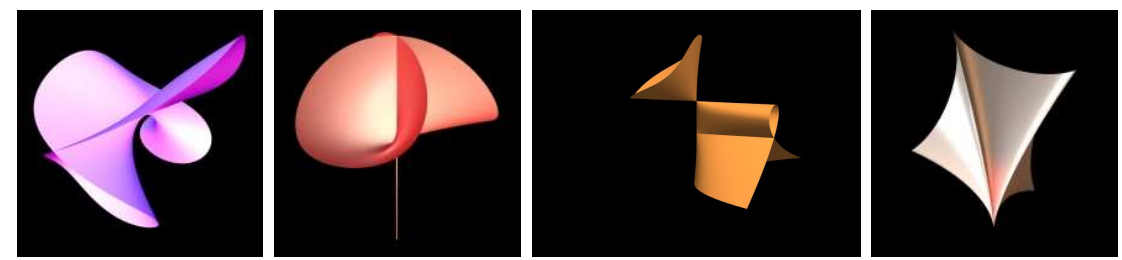

Figures 5, 6, 6' and 7 . 


\section{CLASS 2: Blowups}

For convenience, we restrict to blowups of affine space $\mathbb{A}^{n}$ whose centers are regular closed subschemes $Z$ of $\mathbb{A}^{n}$. All constructions extend naturally to arbitrary regular ambient schemes and centers therein.

The center $Z$ is defined in $\mathbb{A}^{n}$ by an ideal $I_{Z}$ of $K\left[x_{1}, \ldots, x_{n}\right]$, for which we may choose generators $g_{1}, \ldots, g_{k} \in K[x]$. Consider then the map

$$
\gamma: \mathbb{A}^{n} \backslash Z \rightarrow \mathbb{P}^{k-1}: a \rightarrow\left(g_{1}(a): \ldots: g_{k}(a)\right)
$$

where $\left(u_{1}: \ldots: u_{k}\right)$ denote projective coordinates in $\mathbb{P}^{k-1}$. The graph $\Gamma$ of $\gamma$ lives in $\left(\mathbb{A}^{n} \backslash Z\right) \times \mathbb{P}^{k-1}$. We define $\tilde{\mathbb{A}}^{n}$, the blowup of $\mathbb{A}^{n}$ in $Z$, as the Zariski closure of this graph

$$
\tilde{\mathbb{A}}^{n}=\bar{\Gamma} \subset \mathbb{A}^{n} \times \mathbb{P}^{k-1} .
$$

It comes with a natural projection $\Pi: \tilde{\mathbb{A}}^{n} \rightarrow \mathbb{A}^{n}$, the blowup map, induced from the projection $\mathbb{A}^{n} \times \mathbb{P}^{k-1} \rightarrow \mathbb{A}^{n}$ on the first $n$ components (cf. fig. 8) . Different choices of the generators of $I_{Z}$ yield isomorphic blowups. The preimage $Y^{\prime}=\Pi^{-1}(Z)$ is a hypersurface in $\tilde{W}$ called the exceptional divisor. Letting $\left(u_{1}: \ldots: u_{k}\right)$ denote projective coordinates in $\mathbb{P}^{k-1}$, the equations of $\tilde{\mathbb{A}}^{n}$ in $\mathbb{A}^{n} \times \mathbb{P}^{k-1}$ are

$$
u_{i} g_{j}(g)-u_{j} g_{i}(x)=0 \text { for all } i \text { and } j .
$$

We may cover projective space $\mathbb{P}^{k-1}$ by $k$ affine charts isomorphic to $\mathbb{A}^{k-1}$ and given by $u_{j} \neq 0$ for $j=1, \ldots, k$. This, in turn, yields a covering of $\tilde{\mathbb{A}}^{n}$ by $k$ affine charts isomorphic to $\mathbb{A}^{n}$, so that the chart expressions of $\Pi$ can be read off as polynomial maps from $\mathbb{A}^{n}$ to $\mathbb{A}^{n}$. It will always be this description we use to carry out computations and proofs.

If $Z$ is a coordinate subspace, defined by, say, $x_{j}, j \in J$, for some subset $J$ of $\{1, \ldots, n\}$ (this can always be achieved locally after passing to completions), the chart expression in the $j$-th chart is

$$
\begin{aligned}
\Pi_{j}: \mathbb{A}^{n} \rightarrow \mathbb{A}^{n}: x_{i} & \rightarrow x_{i} \text { for } i \notin J \backslash j, \\
x_{i} & \rightarrow x_{i} x_{j} \text { for } i \in J \backslash j .
\end{aligned}
$$

There are several other ways to define blowups, e.g. by a universal property or as the Proj of the Rees algebra associated to the ideal of $Z$. See the 


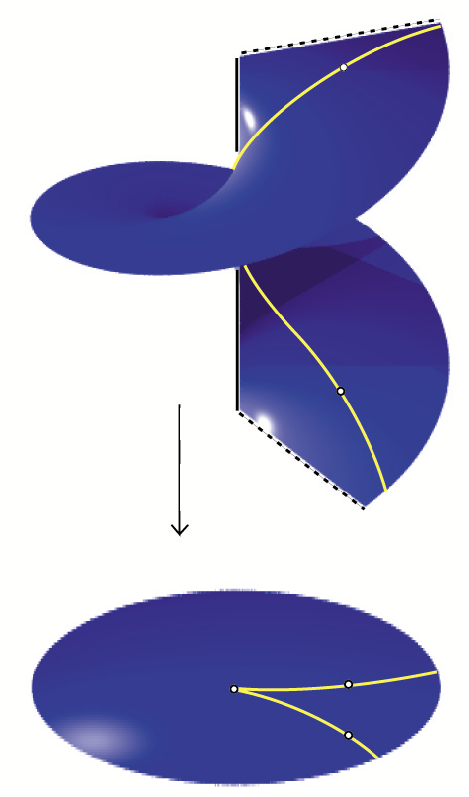

Figure 8: The blowup of $\mathbb{A}^{2}$ in the origin.

lectures [65] or the book of Eisenbud and Harris [54] for more details.

Properties of blowups:

- They are proper birational maps.

- They induce an isomorphism over the complement of the center.

- Blowups commute with localization, completion, restriction to (open or closed) subschemes containing the center (make precise what is meant here - you will have to take the strict transform of the subscheme).

- If $W_{1}=W \times L$ with $L$ regular, the blowup of $W_{1}$ in $Z_{1}=Z \times L$ is the cartesian product of the blowup of $W$ in $Z$ with the identity on $L$.

- Compositions of blowups are again blowups. There is a procedure by G. Bodnár to determine an appropriate center whose blowup yields the composition; it is defined by a non-reduced ideal.

- Local blowups $\left(\tilde{W}, a^{\prime}\right) \rightarrow(W, a)$ (specify what shall mean "local") 
admit coordinates in $W$ at $a$ which make the map monomial. (What happens if $a^{\prime}$ moves along $Y^{\prime}$, how must the coordinates change?)

- If $Z$ is regular and transversal to $X \subset W$ regular or normal crossings (in the sense of the exercises below) then the total transform $X^{*}$ is a scheme with at most normal crossings. If $X$ is regular, the strict transform $X^{s}$ is again regular (and transversal to $Y^{\prime}$ ).

- The ideal $I^{*}$ of the total transform $X^{*}=\Pi^{-1}(X)$ of $X$ in $\tilde{W}$ factors into $I^{*}=I_{Y^{\prime}}^{o} \cdot I^{\curlyvee}$ for a certain ideal $I^{\curlyvee}$ in $\tilde{W}$ (the weak transform of $I$ ) where $o$ denotes the order of $X$ along $Z$ in $W$ (see the next section). Here, $o$ is the maximal power with which $I_{Y^{\prime}}$ can be factored from $I^{*}$.

Remarks. Blowups with regular centers provide a simple algebraic modification of regular schemes $W$ and their singular subschemes $X$, being just a monomial substitution of the variables. Heuristically speaking, the blowup reveals the shape of $X$ along $Z$ up to the first available order of the Taylor expansion. By this we mean the following:

Example. Consider the line $L: y=0$ in $\mathbb{A}^{2}$ and the tangent $k$-th order parabola $P: y=x^{k}$. Both meet at the origin with multiplicity $k$ (i.e., the intersection is a $k$-fold zero). Blowing up the origin, the $y$-chart with map $(x, y) \rightarrow(x y, x)$ is the relevant one (explain why). There, the strict transforms of $L$ and $P$ have equations $y=0$ and $y=x^{k-1}$, so the order of tangency has decreased by 1 .

This shows that blowups in regular centers are a very rough device to resolve singularities. They take into account only a small portion of the geometry of $X$. There exist other modifications, for instance the Nash modification or normalization, which are somewhat more sophisticated procedures. However, they lack some of the basic algebraic features blowups have and which make them so useful.

Exercises: (1) Show that if $Z$ is a regular hypersurface, the blowup map $\Pi$ is an isomorphism.

(2) Show that for regular centers $Z$ in $\mathbb{A}^{n}$, the blowup $\tilde{\mathbb{A}}^{n}$ is again regular and of dimension $n$.

(3) Determine explicitly the covering of $\tilde{\mathbb{A}}^{n}$ by affine charts and the corresponding chart expressions of the blowup map. Then express it in terms of the respective coordinate rings as a certain ring extension. 
(4) Show that if $W$ is a cartesian product $W_{1} \times Z$ and $a$ a point in $Z$, the blowup $\tilde{W} \rightarrow W$ of $W$ in $Z$ is the cartesian product of the blowup $\tilde{W}_{1} \rightarrow W_{1}$ of $W_{1}$ in $Z_{1}=\{a\}$ with the identity on $Z$. Then make the explicit local computations of the blowup of a circle in $\mathbb{A}^{3}$.

(5) Two (or several) schemes are called transversal at a point $a$ if the product of their ideals is a monomial ideal (locally at $a$, with respect to suitable formal coordinates). Take three regular surfaces in $\mathbb{A}^{3}$ so that each two meet transversally. Show that all three need not meet transversally. What happens if you require in addition that all possible intersections of two of the schemes meet transversally?

(6) Consider the blowups $\tilde{W}$ and $\tilde{U}$ of $W=U=\mathbb{A}^{3}$ in the two centers $Z$ and $Z_{1}$ of ideals $(x y, z)$, respectively $(x y, z)(x, z)(y, z)$. What do you observe? Then apply a second blowup (with center a point of your choice) to $\tilde{W}$ and show that the composition equals the blowup $\tilde{U} \rightarrow U$ (provided that you have chosen the correct point on $\tilde{W}$ ).

(7) Define and compute the strict transform of a plane vector field under the blowup of $\mathbb{A}^{2}$ in a point. Do you always get a vector field on $\tilde{\mathbb{A}}^{2}$ ?

(8) Blow up the Fanfare $x^{2}+y^{2}=z^{3}$ in $\mathbb{A}^{3}$ once with center the origin and once with center the $z$-axis. Compute the orders of the respective strict transforms.

(9) Show that the blowup of $\mathbb{A}^{2}$ with center the non-reduced origin of ideal $\left(x, y^{k}\right)\left(x, y^{k-1}\right) \cdots(x, y)$ gives a regular scheme $\tilde{\mathbb{A}}^{2}$ and separates the two components of $x\left(x-y^{k}\right)=0$. Interpret this blowup as a composition of blowups in regular (reduced) centers.

(10) What are the total and strict transforms of a regular hypersurface $X$ in $W$ if the center equals $X$ ? 


\section{CLASS 3: Transforms}

Throughout, $\pi: W^{\prime} \rightarrow W$ denotes the blowup of a scheme $W$ in a regular center $Z$ with exceptional divisor $Y^{\prime}=\pi^{-1}(Z) \subset W^{\prime}$. We shall describe various ways how to lift schemes and ideals in $W$ to schemes and ideals in $W^{\prime}$. Again, we shall stick to an affine scheme $W=\mathbb{A}^{n}$ with coordinate ring $\mathbb{K}[x]=\mathbb{K}\left[x_{1}, \ldots, x_{n}\right]$ and even work locally at a chosen point $a$ of $W$ - taken to be the origin of $\mathbb{A}^{n}$, so that we may argue in the formal power series ring $\mathbb{K}[[x]]=\mathbb{K}\left[\left[x_{1}, \ldots, x_{n}\right]\right]$. By $a^{\prime}$ we shall always denote a point in $Y^{\prime}$ mapping under $\pi$ to $a$. Choosing the local coordinates $x_{1}, \ldots, x_{n}$ suitably at $a$ we may assume that $a^{\prime}$ is the origin of one of the affine charts on $W^{\prime}=\tilde{\mathbb{A}}^{n}$ and the respective chart expression of the blowup map is given by an algebra-homomorphism $\varphi: \mathbb{K}[x] \rightarrow \mathbb{K}[x]$ sending $x_{i}$ to either $x_{i}$ or $x_{i} x_{j}$ as specified earlier (and certainly proven by you in the exercises).

Let $X$ be closed in $W$ and given by the ideal $J$ of $K[x]$ ( $X$ need not be reduced, but we assume that $X$ is rare in $W$, i.e., not equal to one or several components of $W)$. The total transform $X^{*}$ of $X$ is the pullback $\pi^{-1}(X)$ of $X$ in $W^{\prime}$ under $\pi$. Thus, locally at $a^{\prime}$, its ideal equals $J^{*}=\varphi(J)=(f \circ \pi, f \in$ $J$ ). If $Z \subset X$ then $X^{*}$ contains $Y^{\prime}$ as a component (because $X \subsetneq W$ locally at all points). As $Y^{\prime}$ is a hypersurface, we get a factorization $J^{*}=M^{\prime} \cdot I^{\prime}$, where $M^{\prime}$ is a suitable power of the principal ideal $I_{Y^{\prime}}$ defining $Y^{\prime}$ in $W^{\prime}$, say $M^{\prime}=I_{Y^{\prime}}^{o}$ for some $o>0$. The maximal power $o$ of $I_{Y^{\prime}}$ which can be factored from $J^{*}$ is given by the behavior of $X$ along $Z$. More precisely:

The order $\operatorname{ord}_{Z} X$ of $X$ along $Z$ is defined as the maximal integer $k$ so that $J \subset I_{Z}^{k}$. In particular, if $Z=\{a\}$ is a (reduced) point and $X$ is a hypersurface $f=0$, say $J=(f)$, then $\operatorname{ord}_{a} X$ is just the order of vanishing of $f$ at $a$, i.e., the order of the Taylor expansion of $f$ at $a$. If $X$ is not a hypersurface, the order equals the minimum of the orders at $a$ of a generator system of the defining ideal of $X$. Of course, it depends only on the stalk of $J$ at $a$, and the order remains the same when passing to completions. Note that the order depends on the embedding of $X$ in $W$ at $a$. If $X$ is not minimally embedded locally at $a$, (i.e., the dimension of $W$ at $a$ is not minimal among all local embeddings of $X$ at $a$ in a regular ambient scheme) the order of $X$ at $a$ is 1 . In this case, the order is not significant for describing the complexity of the singularity of $X$ at $a$.

For $c \in \mathbb{N}$, we let $\operatorname{top}(X, c)$ be the locus of points where the order of $X$ in $W$ is at least $c$. By the upper semicontinuity of the order, the top locus is a closed (reduced) subscheme. We let $\operatorname{top}(X)$ be the locus of points where the order of $X$ in $W$ is maximal. Of course, we can also define top $(X)$ locally at a point $a$, as the local subscheme where the order of $X$ equals $\operatorname{ord}_{a} X$. 
With these definitions we get the factorization of the total transform $J^{*}=$ $M^{\prime} \cdot I^{\prime}$, where $M^{\prime}=I_{Y^{\prime}}^{o}$ for $o=\operatorname{ord}_{Z} X=\operatorname{ord}_{Z} I$. This order is the maximal power with which $I_{Y^{\prime}}$ can be factored from $J^{*}$. We call $X^{\curlyvee}$ and $J^{\curlyvee}=$ $I_{Y^{\prime}}^{-o} \cdot J^{*}$ the weak transform of $X$ and $J$ under the blowup $\pi: W^{\prime} \rightarrow W$. If $X$ is a hypersurface, it coincides with the strict transform $X^{s}$ of $X$.

One of the basic facts for allowing resolution in the spirit of Hironaka is the following: If the center $Z$ is contained in the top locus of $X$, the order of the weak transform $X^{\curlyvee}$ at points of $Y^{\prime}$ is less or equal the order of $X$ along $Z$,

$$
\operatorname{ord}_{a^{\prime}} X^{\curlyvee} \leq \operatorname{ord}_{a} X .
$$

This holds also for the strict transform (as a consequence of the inequality), and for the Hilbert-Samuel function of $X$ at points $a$, requiring that it is constant along $Z$ and taking a natural ordering among all Hilbert-Samuel functions (see Bennett's paper [22] or [80], [71]).

Properties: As blowups did, passing to the weak transform commutes with restriction to open subschemes, localization and completion. Also, if $X$ and $Z$ are invariant under a group action, the group action lifts to $X^{\curlyvee}$. There are three algebraic properties of weak transforms which we will use repeatedly.

If $P$ and $Q$ are ideals in $W$, we have $(P \cdot Q)^{\curlyvee}=P^{\curlyvee} \cdot Q^{\curlyvee}$. However, $(P+Q)^{\curlyvee} \neq P^{\curlyvee}+Q^{\curlyvee}$ in general, it suffices to take two principal ideals of different order along $Z$. If $\operatorname{ord}_{Z} P=\operatorname{ord}_{Z} Q$, the the weak transform is distributive, say $(P+Q)^{\curlyvee}=P^{\curlyvee}+Q^{\curlyvee}$. There is a nice trick to achieve this equality also in case $p=\operatorname{ord}_{Z} P \neq \operatorname{ord}_{Z} Q=q$. Replace $P+Q$ by the weighted sum $P^{q}+Q^{p}$ and get $\left(P^{q}+Q^{p}\right)^{\curlyvee}=\left(P^{q}\right)^{\curlyvee}+\left(Q^{p}\right)^{\curlyvee}$. As we have $\operatorname{ord}_{a} P^{q}=q \cdot \operatorname{ord}_{a} P$, we do not lose information on the order when passing to powers of ideals.

The third commutation property of weak transforms is with respect to coefficient ideals. These play a decisive role in the induction on the dimension as they allow to pass to ideals in less variables. Their definition is somewhat cumbersome. Let $W=\mathbb{A}^{n}$ with local coordinates $\left(x_{n}, \ldots, x_{1}\right)$ at $a$. For simplicity, we take $a=0$. Let $V$ be the hypersurface in $W$ defined by $x_{n}=0$. Let $I$ be an ideal in $W$ at $a$ of order $o=\operatorname{ord}_{a} I$. The coefficient ideal of $I$ at $a$ in $V$ is defined as the ideal in $V$ generated by certain powers of the coefficients of the elements of $I$ when expanding these with respect to $x_{n}$. More precisely, write $f=\sum_{i \geq 0} a_{i, f}\left(x^{\prime}\right) x_{n}^{i}$ for $f \in I$ and 
with $x^{\prime}=\left(x_{n-1}, \ldots, x_{1}\right)$. Then

$$
\operatorname{coeff}_{V}(I)=\left(\left(a_{i, f}, f \in I\right)^{\frac{o}{o-i}}, i<o\right) .
$$

For example, if $f(x)=x_{n}^{o}+g\left(x^{\prime}\right)$ has no mixed terms, we get coeff ${ }_{V}(f)=$ $(g)$. You will object that, in general, the exponents $\frac{o}{o-i}$ are rational numbers. This can be remedied by taking instead as exponents $\frac{o !}{o-i}$, producing for $f(x)=x_{n}^{o}+g\left(x^{\prime}\right)$ the ideal $\left(g^{(o-1) !}\right)$. Taking factorials loadens the notation without improving the understanding, so we will allow rational exponents and leave it to the reader to define the correct equivalence relation on rational powers of ideals in order to circumvent any traps. As the order of ideals is just multiplied with a constant when passing to powers of ideal, there is no harm in having rational exponents (once you got used to it).

Let $Z \subset \operatorname{top}(I)$ be the center of the blowup $\pi: W^{\prime} \rightarrow W$, with weak transform $I^{\curlyvee}$. Let $a \in Z$ be a point, $V \subset W$ a local hypersurface of $W$ at $a$ (i.e., defined in a neighborhood of $a$ ), let $a^{\prime} \in Y^{\prime}$ be a point in $Y^{\prime}$ so that $a^{\prime}$ lies above $a$ and in the strict (= weak) transform $V^{\prime}$ of $V$. We already know that $\operatorname{ord}_{a^{\prime}} I^{\curlyvee} \leq \operatorname{ord}_{a} I$. If $\operatorname{ord}_{a^{\prime}} I^{\curlyvee}<\operatorname{ord}_{a} I$ we are happy because something has improved. If equality holds (we then say that $a^{\prime}$ is an equiconstant point for $I$ ), we have at least the following commutativity relation

The coefficient ideal of $I^{\curlyvee}$ at $a^{\prime}$ in $V^{\prime}$ is the transform of the coefficient ideal of $I$ at a in $V$,

$$
\operatorname{coeff}_{V^{\prime}} I^{\curlyvee}=\left(\operatorname{coeff}_{V} I\right)^{!} .
$$

This equality does not hold if the order has dropped, and it neither holds if we take on the right hand side the weak transform $\left(\operatorname{coeff}_{V} I\right)^{\curlyvee}$ of $\operatorname{coeff}_{V} I$. Instead, we have to take a new transform, the so called controlled transform. Let $c=\operatorname{ord}_{Z} I$ and define $\left(\operatorname{coeff}_{V} I\right)^{!}=I_{Y^{\prime} \cap V^{\prime}}^{-c} \cdot\left(\operatorname{coeff}_{V} I\right)^{*}$ (the number $c$ is called the control). This is not hard to prove after passing to local coordinates, using that we always have $\operatorname{ord}_{a} \operatorname{coeff}_{V} I \geq \operatorname{ord}_{a} I$. The magic formula with the controlled transform of the coefficient ideal allows to compare ideals in smaller dimension precisely in the case where the order of the original ideal $I$ could not tell us that the singularities improved under blowup. This output recompenses by far the lack of elegance we had to accept in the definition of coefficient ideals.

Be careful: The coefficient ideal nor its order are intrinsic objects. We will have to make an effort to extract coordinate independent information from 
them.

Exercises: (1) Show that $\operatorname{ord}_{Z} X=\min _{a \in Z} \operatorname{ord}_{a} X$ and that $\operatorname{ord}_{a} X$ defines an upper semicontinuous function on $W$. Look up in Hironaka's or Bennett's Annals papers [77], [22] why it does not increase under localization. (This holds also for the Hilbert-Samuel function of $X$ at $a$.)

(2) Compute for several schemes $X$ in $W$ the order of $X$ along a subscheme $Z$ of $W$. Then determine for each $X$ the stratification of $X$ by the strata of constant order (with respect to points).

(3) If $X$ is not locally minimally embedded in $W$ at a point $a \in X$, the order of $X$ at $a$ equals 1 .

(4) Try to find (natural) equations for the top $\operatorname{locus} \operatorname{top}(X, c)$, first in characteristic 0 (easy), then in arbitrary characteristic. In the first case, show that $\operatorname{top}(X)$ lies locally in a regular hypersurface of $W$.

(5) In $J^{*}=M^{\prime} \cdot I^{\prime}$ the order $o=\operatorname{ord}_{Z} X=\operatorname{ord}_{Z} I$ is the maximal power with which $I_{Y^{\prime}}$ can be factored from $J^{*}$.

(6) Let $X$ be a subscheme of $W$ of codimension at least 2, with strict and weak transforms $X^{s}$ and $X^{\curlyvee}$ under the blowup $\pi: W^{\prime} \rightarrow W$. Figure out in three examples which components of $X^{\curlyvee}$ do not show up in $X^{s}$.

(7) (Mandatory) Show that the order of an ideal $I$ in $W$ does not increase when passing to its weak transform, provided the center is included in top $(I)$. Hint: You may work locally in the completion, rectifying thus the center to a coordinate subspace, and then choose coordinates for which the local blowup $\left(W^{\prime}, a^{\prime}\right) \rightarrow(W, a)$ is monomial. (In exceptional cases you are allowed to consult [70] to convince you that it would have been easy.)

(8) Determine in three examples the equiconstant points of an ideal $I$ under blowup, i.e., the points $a^{\prime} \in Y^{\prime}$ where the order of $I^{\curlyvee}$ has remained constant.

(9) Compute the coefficient ideals of $f=x^{3}+y z^{2}$ and $f=x\left(y^{7}-z^{8}\right)$ at 0 with respect to the three coordinate hypersurfaces. Compare the respective orders of the resulting ideals.

(10) Compute the coefficient ideals of the polynomials of (9) after blowing up the origin and compare them with the controlled transforms of the coefficient ideals below. Then prove the commutativity of the passage to coefficient ideals with blowups at equiconstant points. 


\section{CLASS 4: Construction of Mobiles}

In this section we wish to guide you towards the correct definition of mobiles. They shall be intrinsic, globally defined objects at a certain stage of the resolution process containing all information we need in order to define the local resolution invariant and to choose the center of the next blowup. In the last section we shall give the precise definition of mobiles and show how they transform under the blowup with the chosen center. This, in turn, will be used to compute the local invariant after blowup and to show that it decreases at each point of the new exceptional component.

So let us start with an ideal sheaf $\mathcal{J}$ on our regular ambient scheme $W$. We choose a point $a \in W$ and let $J$ denote the stalk of $\mathcal{J}$ at $a$. Taking an affine neighborhood of $a$ in $W$ we may simply assume that $J$ is an ideal of polynomials in $n$ variables with coefficients in the ground field $\mathbb{K}$.

Mobiles control two features of the resolution process: The factorization of ideals into a monomial and a singular part (the exceptional and the not yet resolved portion of the ideal), and the transversality of the chosen centers with the respective exceptional locus. The first task is accomplished by the combinatorial handicap $D$ of the mobile, the second by the transversal handicap $E$. It is appropriate to introduce them in separate sections.

\section{The combinatorial handicap}

We have already seen that after blowup powers of the exceptional components will be factored from the total transform of the ideals, so in order to keep things systematic (which is not very original but helpful) we write $J=M \cdot I$ with $M=1$ the trivial ideal (the whole local $\operatorname{ring}$ ) and $I=J$.

Here is a nice idea: We proceed as we would know what the center of the first blowup is (you remember: often uniqueness is proven before existence, because then you already know how your object has to look like when you try to construct it). So let $Z$ be a closed regular subscheme of $W$ with induced blowup $\pi: W^{\prime} \rightarrow W$ of $W$ along $Z$ and exceptional divisor $Y^{\prime} \subset W^{\prime}$. We denote by $I_{Y^{\prime}}=I_{W^{\prime}}\left(Y^{\prime}\right)$ the ideal defining $Y^{\prime}$ in $W^{\prime}$. We let $J^{\prime}=J^{*}$ be the total transform (inverse image) of $J$ under $\pi$. Its order will have increased, so that's not a good number to look at. Much more interesting is the weak transform, and, to keep things straight, we denote it by $I^{\prime}=I^{\curlyvee}$. Thus $J^{\prime}=M^{\prime} \cdot I^{\prime}$ with $M^{\prime}=I_{Y^{\prime}}^{\mathrm{ord}_{Z} I}$ a normal crossings divisor (even regular for we have blown up only once). Locally, $M^{\prime}$ is just a power of a variable (the variable defining $Y^{\prime}$ in $W^{\prime}$ ). 
Set now $o=\operatorname{ord}_{Z} I$, let $a$ be a point in $Z$ (outside of $Z$ nothing will happen since $\pi$ is an isomorphism there), and let $a^{\prime}$ be any point in $Y^{\prime}$ above $a$, say $\pi\left(a^{\prime}\right)=a$. Set $o^{\prime}=\operatorname{ord}_{a^{\prime}} I^{\prime}$. The next thing to do is to compare $o^{\prime}$ with $o$. Here we remember the key inequality from earlier sections: If the order of $I$ along $Z$ is constant, in particular if $Z \subset \operatorname{top}(I)$, and hence $o^{\prime}=\operatorname{ord}_{a} I$ for all $a \in Z$, we have $o^{\prime} \leq o$, because $I^{\prime}$ is the weak transform of $I$. In view of this pleasant event ("the order does not increase") we immediately agree to allow only centers inside top $(I)$. "Ah", you respond, "maybe we even have $o^{\prime}<o$ for all $a^{\prime}$ above $a . "$ Then we would be done. - Sorry, this is too optimistic, the equlity $o^{\prime}=o$ may occur and the points where this happens form a closed subscheme of $Y^{\prime}$ (but prove that $o^{\prime}<o$ if $n=1$ and $o>0$ ). These are the equiconstant points of $I$ in $Y^{\prime}$.

Before confronting this situation, we do some book-keeping. We will call $D_{n}$ and $D_{n}^{\prime}$ the (non-reduced) divisors defined by $M$ and $M^{\prime}$ in $W$ and $W^{\prime}$ (of course, $D_{n}=\emptyset$ ). They are globally defined and tell us how to factorize the ideals $J$ and $J^{\prime}$. That is information we will need later on. As we shall soon perform the descent in dimension, we write $J_{n}=M_{n} \cdot I_{n}$ for $J=M \cdot I$ and similarly $J_{n}^{\prime}=J^{\prime}$.

So what shall we do at an equiconstant point $a^{\prime}$ above $a$ where the order of $I_{n}^{\prime}$ has remained constant? Now, generically along $Y^{\prime}$, the order drops. Only a few points admit constant order. We suspect that at an equiconstant point $a^{\prime}$ the ideal $I^{\prime}$ must have a special shape. Possibly we can profit of it. Let us therefore observe what happens in two variables, say plane curves. It is immediately seen that $x^{p}+y^{q}$ with $q \geq p$ has weak transform $x^{p}+y^{q-p}$ (in the relevant chart). If $q<2 p$, the order drops and we are done. If $q \geq 2 p$, the order remains constant. However, the degree of the monomial $y^{q}$ has dropped to $q-p$.

This strongly suggests to associate to $I_{n}$ an ideal in one variable less and to look at its order. This is done via coefficient ideals. Choose locally at $a$ a regular hypersurface $V$. You harshly protest because we agreed to choose never an object ad hoc, everything has to be natural. I respond that we are not interested in $V$, neither in the resulting coefficient ideal, but only in its order. It suffices to make this order independent of the choice of $V$. There are two options: either the minimum of all possible orders, over all choices of $V$, or the maximum.

You will have to convince yourself that the minimum is not significant, it just equals $o$. Therefore we take $V$ so that the order of the coefficient ideal is maximized. Such $V$ 's are called hypersurfaces of weak maximal contact with $I_{n}$ at $a$. They exist, and only in case that $I$ is bold regular, i.e., a 
power of a variable, the resulting order is infinite. In this case we redefine the coefficient ideal to be the trivial ideal 1 .

We stop briefly for book-keeping, setting $W_{n-1}=V$ and $J_{n-1}=$ coeff $_{W_{n-1}} I_{n}$. The letter $J$ is taken instead of $I$ because, as we saw in class II, coefficient ideals do not pass to the weak transform under blowup (the letter $I$ is reserved for ideals which pass to weak transforms). For accurateness, we factorize $J_{n-1}=M_{n-1} \cdot I_{n-1}$ with $M_{n-1}=1$ and $I_{n-1}=J_{n-1}$, and set $o_{n-1}=\operatorname{ord}_{a} I_{n-1}$.

Let's go to $W^{\prime}$ at $a^{\prime}$ and $J_{n}^{\prime}=M_{n}^{\prime} \cdot I_{n}^{\prime}$. Denote by $J_{n-1}^{\prime}$ the coefficient ideal of $I_{n}^{\prime}$ with respect to a local hypersurface $W_{n-1}^{\prime}$ which maximizes its order. The curve case suggests that $J_{n-1}^{\prime}$ has something to do with $J_{n-1}$. We are now curious to explore this connection between the coefficient ideals of $I_{n}$ and $I_{n}^{\prime}$ (we have seen portion of it in class II). Remember that $J_{n-1}^{\prime}$ equals the controlled transform of $J_{n-1}$ if $W_{n-1}^{\prime}$ is the strict transform of $W_{n-1}$ (in particular, $a^{\prime}$ must be included in $W_{n-1}^{\prime}$ ).

At this point, where things seem to become more and more involved, there pop up a few very favorable coincidences. They will make everything work marvellously - provided we are in characteristic 0. Such lucky strokes are rare in mathematics, and I see no substantial reason why they occur precisely here and now. Once Abhyankar and Hironaka discovered them in the fifties (stories tell that the latter was visiting the former and insisted for four days until he had completely clarified the former's vision of using Tschirnhaus' transformation for resolution purposes), the rest was only technique (as other stories tell).

In positive characteristic these coincidences do not occur - and nobody has found a working substitute for them. At least for the arguments and constructions to follow, the characteristic $p$ case is much less accessible, if at all.

Stroke 1: If $W_{n-1}$ maximizes the order of $J_{n-1}=$ coeff $_{W_{n-1}} I_{n}$ at $a$ (everything is local), it contains locally the top locus top $\left(I_{n}\right)$ of $I_{n}$. False in characteristic $p>0$, see [104], [103] or [67].

Stroke 2: There is a simple procedure to construct such hypersurfaces of weak maximal contact (not all), via osculating hypersurfaces, see [55] or [70]. Look up the definition there or see the exercises. This construction appears in various forms in most of the resolution papers. Hypersurfaces of weak maximal contact can also be constructed (by different means) in positive characteristic, but do not enjoy the same nice properties. 
Stroke 3: If $W_{n-1}$ maximizes the order of $J_{n-1}=\operatorname{coeff}_{W_{n-1}} I_{n}$ at $a$, its strict transform $W_{n-1}^{\prime}$ contains all equiconstant points of $I_{n}$ in $Y^{\prime}$. First observed by Zariski. Proof: Computation in local coordinates. Also ok in positive characteristic.

Stroke 4: If $W_{n-1}$ is osculating for $I_{n}$, in particular, maximizes the order of $J_{n-1}=\operatorname{coeff}_{W_{n-1}} I_{n}$ at $a$, and if the order has remained constant, $o_{n}^{\prime}=o_{n}$ at $a^{\prime}$, its strict transform $W_{n-1}^{\prime}$ is osculating for $I_{n}^{\prime}$, in particular, maximizes the order of $J_{n-1}^{\prime}=$ coeff $_{W_{n-1}^{\prime}} I_{n}^{\prime}$ at $a^{\prime}$. Proof: Computation in local coordinates. False in positive characteristic, see [66].

With this gambling things become easy. Fix $a \in Z$ and $a^{\prime} \in Y^{\prime}$ above $a$. Choose $W_{n-1}$ osculating at $a$ for $I_{n}$, let $J_{n-1}$ be the corresponding coefficient ideal of $I_{n}$ in $W_{n-1}$. Then, at each equiconstant point $a^{\prime}$ above $a$, $W_{n-1}^{\prime}=W_{n-1}^{s}$ is osculating for the weak transform $I_{n}^{\prime}=I_{n}^{\curlyvee}$. This ideal has as coefficient ideal $J_{n-1}^{\prime}$ the controlled transform $\left(J_{n-1}\right)^{!}=I_{Y_{n-1}^{\prime}}^{-\operatorname{ord}_{a} I_{n}} \cdot J_{n-1}^{*}$ of $J_{n-1}$ in $W_{n-1}^{\prime}$, where $I_{Y_{n-1}^{\prime}}=I_{W_{n-1}^{\prime}}\left(Y^{\prime} \cap W_{n-1}^{\prime}\right)$ denotes the ideal defining in $W_{n-1}^{\prime}$ the exceptional divisor $Y^{\prime} \cap W_{n-1}^{\prime}$ of the blowup $W_{n-1}^{\prime} \rightarrow W_{n-1}$. Recall here that, locally at $a, Z$ is contained in $W_{n-1}$, so that $Y^{\prime} \cap W_{n-1}^{\prime}$ is regular.

In particular, we may factorize $J_{n-1}^{\prime}=M_{n-1}^{\prime} \cdot I_{n-1}^{\prime}$ with $I_{n-1}^{\prime}=I_{n-1}^{\curlyvee}$ the weak transform of $I_{n-1}$ and $M_{n-1}^{\prime}$ a normal crossings divisor in $W_{n-1}^{\prime}$ supported by the exceptional component $Y^{\prime} \cap W_{n-1}^{\prime}$. Hence the divisor $D_{n-1}^{\prime}=\left(\operatorname{ord}_{a} I_{n-1}-\operatorname{ord}_{a} I_{n}\right) \cdot Y^{\prime}$ of $W^{\prime}$ has normal crossings at $a^{\prime}$ and defines the principal monomial ideal $I_{W_{n-1}^{\prime}}\left(D_{n-1}^{\prime} \cap W_{n-1}^{\prime}\right)=M_{n-1}^{\prime}$ locally at $a^{\prime}$.

This looks a little bit complicated. And indeed, it is complicated, especially, if you are not yet used to this type of constructions. But always keep in mind the corresponding commutative diagram, with vertical arrows the blowups in $W_{n}$ and $W_{n-1}$, and horizontal arrows the descent in dimension. If you draw it for yourself on a sheet of paper things will clarify immediately (after having done one explicit computation for, say, a surface singularity). And you will realize that, again, everything is absolutely systematic.

Let us collect our data at the point $a$ and at the equiconstant point $a^{\prime}$ above $a$ :

$$
\begin{aligned}
& J_{n}=M_{n} \cdot I_{n} \text { in } W_{n}, \\
& J_{n}^{\prime}=M_{n}^{\prime} \cdot I_{n}^{\prime} \text { in } W_{n}^{\prime},
\end{aligned}
$$




$$
\begin{aligned}
& J_{n-1}=M_{n-1} \cdot I_{n-1} \text { in } W_{n-1}, \\
& J_{n-1}^{\prime}=M_{n-1}^{\prime} \cdot I_{n-1}^{\prime} \text { in } W_{n-1}^{\prime} \text {, and } \\
& I_{n}^{\prime} \text { and } I_{n-1}^{\prime} \text { are the weak transforms of } I_{n} \text { and } I_{n-1} .
\end{aligned}
$$

Moreover,

$$
D_{n}^{\prime}=\operatorname{ord}_{a} I_{n} \cdot Y^{\prime} \text { and } D_{n-1}^{\prime}=\left(\operatorname{ord}_{a} I_{n-1}-\operatorname{ord}_{a} I_{n}\right) \cdot Y^{\prime} .
$$

By the way, what are these data at a point $a^{\prime}$ where the order of $I_{n}^{\prime}$ has dropped? Either we refuse to define them, since our induction on the order already works, or, as we shall do, we choose any (new) osculating hypersurface $W_{n-1}^{\prime}$ for $I_{n}^{\prime}$ at $a^{\prime}$, set $J_{n-1}^{\prime}=\operatorname{coeff}_{W_{n-1}^{\prime}}\left(I_{n}^{\prime}\right)$ with trivial factorization $J_{n-1}^{\prime}=1 \cdot I_{n-1}^{\prime}$ (no other factorization need hold). Of course, $I_{n-1}^{\prime}$ is no longer the weak transform of $I_{n-1}$, so that its order may be quite arbitrary, but we don't care, since - lexicographically - the pair $\left(o_{n}^{\prime}, o_{n-1}^{\prime}\right)<\left(o_{n}, o_{n-1}\right)$ has dropped at $a^{\prime}$. You may notice that $D_{n-1}^{\prime}$, though globally defined on $W^{\prime}$, is only a stratified divisor, since the multiplicity of $Y^{\prime}$ depends on the point $a^{\prime}$. Specify what are the strata along which $D_{n-1}^{\prime}$ is coherent?

At this point, you may wish to see a concrete example. Here it is: Let $J=J_{2}$ be the principal ideal in $W=W_{2}=\mathbb{A}^{2}$ generated by $f=x^{p}+y^{q}$ with $0<p \leq q$. We place ourselves at the origin $a=0$ of $\mathbb{A}^{2}$, which is the only singular point of the plane curve $X$ defined by $f$. As no blowup has occured so far, $J_{2}=M_{2} \cdot I_{2}$ with $M_{2}=1$ and $I_{2}=J_{2}$. The order $o_{2}=\operatorname{ord}_{a} I_{2}$ equals 2 . In characteristic 0 , the hypersurface $W_{1}$ defined by $x=0$ in $W_{2}$ maximizes the order of the coefficient ideal $J_{1}=\operatorname{coeff}_{W_{1}}\left(I_{2}\right)=\left(y^{q}\right)$. (If the characteristic equals $p$, this is not true if $q$ is a multiple of $p$.) We get $J_{1}=M_{1} \cdot I_{1}$ with $M_{1}=1$ and $I_{1}=J_{1}$. Clearly, $o_{1}=\operatorname{ord}_{a} I_{1}=q \geq p$.

The invariant is the pair $\left(o_{2}, o_{1}\right)=\left(\operatorname{ord}_{a} I_{2}, \operatorname{ord}_{a} I_{1}\right)$ and attains at $a=0$ its maximal value $(p, q)$. This will therefore be our first center of blowup, $Z=\{0\}$ in $\mathbb{A}^{2}$ with blowup $\pi: W_{2}^{\prime} \rightarrow W_{2}$ and exceptional divisor $Y^{\prime} \subset W_{2}^{\prime}$. Let $a^{\prime}$ be a point of $Y^{\prime}$. If $a^{\prime}$ is the origin of the $x$-chart, the order of $I_{2}^{\prime}=I_{2}^{\curlyvee}=\left(1+x^{q} p^{q}\right)$ has dropped to 0 , so that there $J_{2}^{\prime}=I_{Y^{\prime}}^{p} \cdot 1$ and $W_{1}^{\prime}$ can be chosen arbitrarily, with $J_{1}^{\prime}=1$ (by definition of the coefficient ideal of the trivial ideal). Hence $M_{1}^{\prime}=I_{1}^{\prime}=1$ and the orders are $\left(o_{2}^{\prime}, o_{1}^{\prime}\right)=(0,0)<\left(o_{2}, o_{1}\right)=(p, q)$. The same phenomenon occurs at all point $a^{\prime}$ of $Y^{\prime}$ outside the origin of the $y$-chart.

So let us look at this origin. It is the most interesting point. There, the order $o_{2}^{\prime}$ of $I_{2}^{\prime}=$ is $q-p$ if $q<2 p$ and $p$ if $q \geq 2 p$. In the first case, the order has dropped, $I_{2}^{\prime}=\left(y^{q-p}+x^{p}\right)$ and our local hypersurface $W_{1}^{\prime}$ will 
now be chosen as $y=0$ with coefficient ideal $J_{1}^{\prime}=\left(x^{p}\right)$. The factorization is $J_{1}^{\prime}=M_{1}^{\prime} \cdot I_{1}^{\prime}$ with $M_{1}^{\prime}=1$ and $I_{1}^{\prime}=J_{1}^{\prime}$. You see that $I_{1}^{\prime}$ is not the weak transform of $I_{1}^{\prime}$, which does not matter because $o_{2}^{\prime}$ has dropped so that the pair of orders $\left(o_{2}^{\prime}, o_{1}^{\prime}\right)=(q-p, p)$ has dropped lexicographically.

We are left with the case $q \geq 2 p$. The order of $I_{2}^{\prime}=\left(x^{p}+y^{q-p}\right)$ at $a^{\prime}$ (the origin of the $y$-chart) has remained constant equal to $p$. Therefore we will really need the descent in dimension here. The local hypersurface $W_{1}^{\prime}$ can be chosen equal to the strict transform $W_{1}^{s}$ of $W_{1}$. It has equation $x=0$ in this chart. The coefficient ideal $J_{1}^{\prime}$ is generated by $y^{q-p}$ and factorizes into $J_{1}^{\prime}=M_{1}^{\prime} \cdot I_{1}^{\prime}$ with $I_{1}^{\prime}=1$ the weak transform of $I_{1}=\left(y^{q}\right)$ under the blowup of $W_{1}$ in $Z=\{0\}$. Hence $M_{1}^{\prime}=J_{1}^{\prime}=\left(y^{q-p}\right)$. As for the orders at $a^{\prime}$, we get $\left(o_{2}^{\prime}, o_{1}^{\prime}\right)=(p, 0)$ which is lexicogaphically smaller than $\left(o_{2}, o_{1}\right)=(p, q)$. Our induction is thus completed at all points $a^{\prime}$ of $Y^{\prime}$.

To make things more explicit, we write down the two combinatorial handicaps before and after blowup. In $W=W_{2}$ we have $D=\left(D_{2}, D_{1}\right)=(\emptyset, \emptyset)$ everywhere. If $q<2 p$, the combinatorial handicap $D^{\prime}$ in $W^{\prime}=W_{2}^{\prime}$ equals everywhere $\left(D_{2}^{\prime}, D_{1}^{\prime}\right)=\left(p \cdot Y^{\prime}, \emptyset\right)$. If $q \geq 2 p$, we stratify $W_{2}^{\prime}$ into $S=W_{2}^{\prime} \backslash\left\{0_{y \text {-chart }}\right\}$ and $T=\left\{0_{y \text {-chart }}\right\}$. At all points of $S$ we have $D_{2}^{\prime}=p \cdot Y^{\prime}$ and $D_{1}^{\prime}=\emptyset$. In contrast, at the origin of the $y$-chart we have $D_{2}^{\prime}=p \cdot Y^{\prime}$ and $D_{1}^{\prime}=(q-p) \cdot Y^{\prime}$, so that indeed $M_{2}^{\prime}=\left(y^{p}\right)$ in $W_{2}^{\prime}$ and $M_{1}^{\prime}=\left(y^{q-p}\right)$ in $W_{1}^{\prime}$. Notice here that $W_{1}^{\prime}$ defined by $x=0$ is transversal to $Y^{\prime}$.

Exercises: (1) Prove that $o^{\prime}<o$ if $n=1$ and $o>0$, for $o=\operatorname{ord}_{a} I$ and $o^{\prime}=\operatorname{ord}_{a^{\prime}} I^{\prime}$, taking $I^{\prime}=I^{\curlyvee}$ the weak transform. Hint: Determine first the center $Z$.

(2) Figure out why the four lucky strokes hold in characteristic 0? Look up the counterexamples in positive characteristic.

(3) Show that in characteristic 0, the local top locus of an ideal is contained in a regular hypersurface whose weak transform contains all equiconstant points (this hypersurface will be defined by a suitable derivative of the generators of the ideal). Then look up the example of Narasimhan in positive characteristic (see [70]).

(4) Assume that $q \geq 3 p$ and compute the combinatorial handicap for the plane curve $x^{p}+y^{q}=0$ after the second blowup. What would happen in characteristic $p$ for $q=3 p$ ?

(5) Resolve the Whitney-umbrella $x^{2}+y z^{2}=0$ by taking as center the top 
locus of the triple of orders $\left(o_{3}, o_{2}, o_{1}\right)$. If you got tired of the computations, write a program which computes all data.

(6) Do the same for the surface $x^{2}+y^{3}+z^{4}=0$. Then find out why we really need the combinatorial handicap $D$ at all stages of the resolution process and what its transformation rule is.

\section{The transversal handicap}

Assume that we are at a certain stage of our resolution process and wish to make the next blowup. In this section we address the question how to ensure that our chosen center is transversal to the already existing exceptional components which were produced by the preceding blowups. Recall that this transversality is necessary to get after the blowup a new exceptional locus having again normal crossings.

Let us denote $W$ our present ambient scheme, $\mathcal{J}$ the ideal sheaf we wish to resolve, $a$ a point of $W$ and $J$ the stalk of $\mathcal{J}$ at $a$. Let $F$ be the exceptional locus in $W$ produced by the prior blowups. By induction on the number of blowups we may assume that $F$ has normal crossings. As transversality of two schemes is a local property compatible with completion, we may stick to a neighborhood of $a$ in $W$ and pass, if necessary, to the completion of the local rings. Thus we may suppose that $W=\mathbb{A}^{n}$ and that $J$ is a polynomial ideal.

In order to know how to factorize $J$ and the subsequent local coefficient ideals at $a$ into a product of a principal monomial ideal and a remaining factor, we have introduced and constructed in the last section the combinatorial handicap $D$ in $W$. It consists of normal crossings divisors $D_{n}, \ldots, D_{1}$ in $W$ so that $J_{i}=M_{i} \cdot I_{i}$ for all $n \geq i \geq 1$, where $M_{i}=I_{W_{i}}\left(D_{i} \cap W_{i}\right)$ are the ideals associated to a local flag of regular schemes $W=W_{n} \supset W_{n-1} \supset \ldots \supset W_{1}$ at $a$.

Neglecting transversality problems, the center of blowup would be, locally at $a$, the scheme $W_{d-1}$ with $d$ minimal so that $I_{d} \neq 1$ (then $W_{d-1}=\operatorname{top}\left(I_{d}\right)$ is just the support of $I_{d}$ ). Despite the fact that the flag $W_{n} \supset \ldots \supset W_{1}$ is not intrinsic (there are many possible choices), we saw that the so defined center does not depend on these choices and gives a global closed and regular subscheme of $W$. Let us call it the virtual center $Z^{\text {virt }}$. Virtual, because, in practice, the actual center $Z=Z^{a c t u}$ of the next blowup will mostly be different from $Z^{\text {virt }}$ (it will be contained in $Z^{\text {virt }}$ ), precisely for transversality reasons with the exceptional locus $F$. 
So let us investigate the precise constellation of $Z^{\text {virt }}$ and $F$. Again, the question is local. We may assume that the point $a$ lies in the intersection of both, otherwise $Z^{\text {virt }}$ and $F$ are trivially transversal at $a$. At an intersection point $a$, several things may happen. Recall here that we consider two schemes to be transversal at $a$ if the product of their ideals in $W$ defines a normal crossings scheme (i.e., if the ideal generated by the product in the completion of the local ring of $W$ at $a$ can be generated by monomials). If $Z^{\text {virt }}$ is contained in all components of $F$ passing through $a$ (the intersection of these components is just the local top locus of $F$ at $a$ ), it is certainly transversal to $F$. If it is not contained, it may be transversal to some components of $F$ and not transversal to others. In this case, we will have to choose a smaller center $Z$ inside $Z^{\text {virt }}$. But which one? Taking simply for $Z$ the intersection of $Z^{\text {virt }}$ with all components of $F$ to which it fails to be transversal does not work because this intersection will in general be singular scheme-theoretically.

Sticking to our philosophy from earlier sections, we proceed again upside down and assume that we already know how to choose the actual center $Z^{a c t u}$ transversal to $F$. This is not a bad idea, but once in a while we will have to stop waving hands and to start making Nägel mit Köpfen. In any case, let's see what happens.

If $Z^{a c t u}=Z^{\text {virt }}$, everything is fine, our invariant introduced (vaguely) in the section on the combinatorial handicap will drop (this will be explicited more carefully in the last section) and (vertical) induction applies. By transversality of $Z$ with $F$, the new exceptional divisor $F^{\prime}$ in $W^{\prime}$ will have again normal crossings. Fine!

So let us look at the case $Z^{a c t u} \subsetneq Z^{\text {virt }}$. Something surprising is happening (in retrospection, it won't be such a surprise): The resolution invariant remains constant, the situation seems not to improve. Why is this the case? The clue is the upper semicontinuity of the invariant: By construction, it is constant along $Z^{\text {virt }}$ and attains its maximal value there $\left(Z^{\text {virt }}\right.$ is the top locus of the invariant). Along the open subscheme $U=Z^{v i r t} \backslash Z^{a c t u}$ of $Z^{\text {virt }}$ the blowup is a local isomorphism, so at points of the strict transform $U^{s}$ the invariant will remain constant. By upper semicontinuity, it has the same value on the closure $\bar{U}$ in $W^{\prime}$. As this closure meets the new exceptional component $Y^{\prime}=\pi^{-1}\left(Z^{a c t u}\right)$, there will be points of $Y^{\prime}$ where the invariant has not dropped. We are stuck.

We suggest that you digest briefly this last paragraph by taking for $X$ in $\mathbb{A}^{3}$ the cartesian product of the plane cusp $z^{3}=y^{2}$ with the $x$-axis and for $F$ the the cartesian product of the parabola $y=x^{2}$ with the $z$-axis. The 
virtual center $Z^{\text {virt }}$ will be the $x$-axis (make sure that no other choice makes sense), which is tangent to $F$ and hence not allowed as center. Instead, we have to take $Z^{a c t u}=\{0\}$ the origin, and the transform $X^{\prime}$ of $X$ in $W^{\prime}=\widetilde{\mathbb{A}^{3}}$ looks quite the same.

Doing mathematics is - aside genuine Geistesblitze - a Wechselspiel of computing examples; observing; pointing out obstructions; finding the reasons for the obstructions; observing again; trying to isolate the obstruction so as to see clearly its Ursprung; computing once more, etc. Looking carefully at phenomena and complicated configurations is one of the most delicate jobs for mathematicians. Often we just do not see what is there, and of what we could profit of. And only afterwards the solution to the problem seems so natural, so evident. If we had just seen it earlier.

In view of these "profound" philosophical and pedagogical contemplations, we look once again at our situation.

By transversality, we are forced to choose a center smaller than the one we would like to take and which would make the invariant drop. Being too small, the invariant remains the same (at least at some points of the new exceptional component) and our induction breaks down. The invariant is not able to detect any improvement of the singularities.

At this point we will ask ourselves why we blow up at all if it does not help to advance the induction. We could as well do nothing and resignate. This question is precisely the correct one, so we repeat it: Why blowing up at all if the virtual center is not transversal to the exceptional locus.

The question contains, at least in this case, also the answer. We blow up because we wish to improve our resolution problem, which consists in making an ideal a monomial ideal. But our non-transversality problem we encounter on the way is precisely of the same nature as our original problem: An ideal (in this case the product of the ideal of $Z^{\text {virt }}$ and of $F$ ) is not a monomial ideal.

After all this much-talking-and-little-saying it should have become clear what to do: We interpret the non-transversality problem as a separate resolution problem and try to resolve it first in order to be able afterwards once it is solved - to choose indeed the virtual center as the actual center. Therefore our present blowup with center $Z^{a c t u} \subsetneq Z^{\text {virt }}$ has the intention to help to make $Z^{v i r t}$ transversal to $F$. This is the true purpose of the blowup, and obviously the invariant associated to the ideal $J$ won't recognize that. Looking back at the example from before, we see that the blowup does improve the transversality problem, after the blowup the virtual center is 
again the $x$-axis, but the transform $F^{\prime}$ of $F$ is now transversal to the $x$ axis.

This is encouraging and we immediately start to build up the data for our secondary (= transversality) resolution problem. The approach indeed works, though it burdens considerably the whole setting and constructions. Just imagine that along the solution of the subproblem new exceptional components will pop up, and while solving the transversality issue we may confront another transversality problem, which we have to solve first before we are allowed to attack the original one. And so on. This is technically (very) frightening.

There is an elegant solution to this annoying superposition of subordinate resolution problems suggested by Villamayor in [121]. In each step of the descent in dimension via local flags $W_{n} \supset \ldots \supset W_{1}$ take care in advance of the transversality problem by modifying the ideals $I_{i}$ so that the resulting center is already contained in all exceptional components to which the virtual center may not be transversal. Just multiply $I_{i}$ by the ideal $Q_{i}$ of dangerous components, i.e., those to which the next local hypersurface $W_{i-1}$ may not be transversal. Then the top locus of $K_{i}=I_{i} \cdot Q_{i}$ is contained in $W_{i-1}$ locally at $a$.

The dangerous exceptional components are collected in the transversal handicap $E=\left(E_{n}, \ldots, E_{1}\right)$. Here, $E_{i}$ is the normal crossings divisor formed by those exceptional components to which $W_{i-1}$ may not be transversal. Even though $W_{i-1}$ is not intrinsic, $E_{i}$ will not depend on any choices and will obey a precise law of transformation under blowup. We will specify this law in the next section.

Meanwhile, let us see the impact of the construction. First, the components $o_{i}=\operatorname{ord}_{a} I_{i}$ of the invariant will be replaced by pairs $\left(o_{i}, q_{i}\right)$ where $q_{i}=\operatorname{ord}_{a} Q_{i}$ measures the advance of the transversality problem in dimension $i-1$ (the shift by 1 has notational reasons). If the components $\left(o_{n}, q_{n}, o_{n-1}, \ldots, o_{i}\right)$ have remained constant under blowup, the transformation law for $E$ says that $Q_{i}$ passes to its weak transform $Q_{i}^{r}$. As the center will lie in $\operatorname{top}\left(K_{i}\right)=\operatorname{top}\left(I_{i}\right) \cap \operatorname{top}\left(Q_{i}\right)$ (here, the top loci have to be considered locally at $a$ ), the order of $Q_{i}$ won't increase. This immediately implies the fabulous inequality

$$
\left(o_{n}^{\prime}, q_{n}^{\prime}, o_{n-1}^{\prime}, \ldots, o_{i}^{\prime}, q_{i}^{\prime}\right) \leq\left(o_{n}, q_{n}, o_{n-1}, \ldots, o_{i}, q_{i}\right)
$$

where the two vectors are compared lexicographically. And by exhaustion of the dimensions, when looking at the whole new invariant $\left(o_{n}, q_{n}, \ldots, o_{1}, q_{1}\right)$, 
it must have decreased.

There are some technical details which still have to be filled in. For instance, the local hypersurface $W_{i-1}$ at $a$ will be chosen to be osculating for $I_{i}$ (and not, as one may think, for $K_{i}$ ). Also, one has to take care for establishing the necessary inclusions of the various top loci, for expliciting the transformation laws for all the ideals $J_{i}, M_{i}, I_{i}, Q_{i}, K_{i}$, and for ensuring that the resulting center is indeed transversal to the current exceptional locus $F$. All this can be done. Due to the systematic approach, it is even not as breathtaking as one might expect. We will see portion of it in the next section.

The determination of the dangerous exceptional components has a computational drawback. For each $i$, we have to take all possibly non-transversal components of $F$ with respect to $W_{i-1}$, and many of these could already be transversal, but we just don't see it, because our invariant is unable to check it out. This inconvenience increases considerably the complexity of the algorithm. However, concerning the theoretical part of the construction of the resolution, it is quite useful because it follows in each dimension the same pattern and uses only information prescribed by the local invariant. Thus it is automatically intrinsic (i.e., independent of the local choices of hypersurfaces, hence global), and allows a systematic treatment via inductions on the dimensions.

If you look up the paper [55] you will realize that the hardest part is to become familiar with all the constructions and definitions collected in the section Concepts. The purpose of these lecture notes and [70] and [65] is precisely to motivate these constructions and to give you some feeling for them. But then, the actual proofs are rather short and almost routine. See the sections Transversality or Top loci in [55].

Exercises. (1) Two regular subschemes $U$ and $V$ of $W$ meet transversally (in the sense defined above), if and only if their intersection $U \cap V$ is a regular scheme. Does this hold also for three regular subschemes, taking all pairwise intersections? (You may remember an earlier exercise.)

(2) Let $F$ be a normal crossings scheme. Show that all possible intersections of components of $F$ meet transversally. Does the converse hold?

(3) If, locally at a point $a$ in $W$, a regular scheme $Z$ is contained in all components of a normal crossings divisor $F$ passing through $a$, then $Z$ is transversal to $F$ at $a$. 
(4) Assume that a regular scheme $Z$ meets all intersections of the components of a normal crossings scheme $F$ in $W$ transversally. Determine the cases when $Z$ meets $F$ transversally and when not.

(5) In the situation of exercise (4), consider the blowup $W^{\prime} \rightarrow W$ of $W$ with center $Z$, and let $F^{*}$ be the total transform of $F$ in $W^{\prime}$. Is $F^{*}$ again a normal crossings scheme in $W^{\prime}$ ?

(6) Start at zero, i.e., with empty exceptional locus, and blow up once $W=$ $\mathbb{A}^{3}$ at 0 . Figure out whether in $W^{\prime}$ there can already occur a transversality problem, and if yes, determine the dimensions where it becomes virulent. Then indicate the transversal handicap $E^{\prime}=\left(E_{3}^{\prime}, E_{2}^{\prime}, E_{1}^{\prime}\right)$ in $W^{\prime}$. Hint: $E^{\prime}$ will again consist of stratified divisors, the strata being given by the values of the invariant along the new exceptional component $Y^{\prime}$.

(7) If you have done all the exercises up to now you are allowed to take a break. Otherwise return to the last exercise you did not do and give it a new try. 


\section{CLASS 5: Resolution of Mobiles}

Here is now the precise definition of mobiles. A singular mobile on a regular $n$-dimensional ambient scheme $W$ is a quadruple $\mathcal{M}=(\mathcal{J}, c, D, E)$ where $\mathcal{J}$ is a coherent ideal sheaf on $W$ (one could also allow $\mathcal{J}$ to live on a regular, locally closed subscheme $V$ of $W$, cf. [55]), $c$ is a positive integer, the control, and $D=\left(D_{n}, \ldots, D_{1}\right)$ and $E=\left(E_{n}, \ldots, E_{1}\right)$ are strings of stratified normal crossings divisors $D_{i}$ and $E_{i}$ on $W$. Stratified means that there is a finite stratification of $W$ by locally closed subschemes such that each $D_{i}$ and $E_{i}$ is coherent along the strata.

We call $D$, respectively $E$, the combinatorial and transversal handicap of $\mathcal{M}$. The divisors $D_{i}$ are in general not reduced; they carry a small additional information, their label, which allows to order the components of $D_{i}$, but which shall not bother us here (for details, see [55]). The divisors $E_{i}$ are reduced, have no components in common, and their union $|E|$ will equal the exceptional locus in $W$ at the current stage of the resolution process. You should think of a mobile as follows (cf. the last chapter): The ideal $\mathcal{J}$ is the ideal defining the singular scheme $X$ in $W$ we wish to resolve. It passes under blowup to its controlled transform $\mathcal{J}^{!}=I\left(Y^{\prime}\right)^{-c} \cdot J^{*}$ with respect to c. At the beginning, the handicaps are trivial, $D_{i}=E_{i}=\emptyset$. Under blowup, they obey a precise law of transformation, which we shall describe later on. This will allow to associate to any mobile $\mathcal{M}$ in $W$ and blowup $W^{\prime} \rightarrow W$ the transformed mobile $\mathcal{M}^{\prime}$ in $W^{\prime}$. We say that the mobile $\mathcal{M}$ is resolved, if the order of $\mathcal{J}$ at all points of $W$ is less than $c$. Notice here that for $c=2$ and $\mathcal{J}$ a principal ideal, this signifies that $\mathcal{J}$ defines a regular scheme. However, as the order is not so significant for non-hypersurfaces (order 1 at a point just means that the scheme is locally not minimally embedded in the ambient scheme), it is more convenient to take the control $c=1$, in which case the mobile is resolved if $\mathcal{J}$ is the structure sheaf of $W$. This, in turn, signifies that the scheme we started with has as total transform a normal crossings divisor.

Mobiles are not as complicated as one might think. They are globally defined objects which do not depend on any ad hoc or local choices. The delicate part is to associate to them a local invariant and to define the transformation law. These two things are strongly related to each other. In the course of their definition we will have to consider objects which are not intrinsic and only locally defined. But we don't care as long as the final output is intrinsic.

For a mobile $\mathcal{M}$ and a point $a$ in $W$, the local invariant $i_{a}(\mathcal{M})$ of $\mathcal{M}$ at $a$ will be a vector of integers, and these integers are the orders of certain ideals 
defined locally at $a$. Thus we have perfect control on them under blowup as long as the ideal in question passes to its weak transform. Namely, in this case, the order of the ideal won't increase. As we have already seen in earlier sections, the respective ideals will indeed pass to their weak transforms provided that the earlier components of our invariant have remained constant. This suggests to consider $i_{a}(\mathcal{M})$ with respect to the lexicographic ordering.

Let us now see the details. We shall associate to $\mathcal{M}$ and $a$ ideals $J_{n}, \ldots, J_{1}$, $I_{n}, \ldots, I_{1}$ and $K_{n}, \ldots, K_{1}$ defined in local flags $W_{n} \supset \ldots \supset W_{1}$ at $a$. The $W_{i}$ are regular hypersurfaces in $W_{i+1}$ defined in a neighborhood of $a$, where $W_{n}=W$ is the ambient scheme. There will be a certain rule how to choose them, but in any case they are not unique nor intrinsic. The ideals $J_{i}, I_{i}$ and $K_{i}$ are defined in $W_{i}$, and are neither intrinsic. We denote them by roman letters, because we think of them as the stalks at $a$ of ideal sheaves. Our invariant is then simply the vector

$$
i_{a}(\mathcal{M})=\left(o_{n}, k_{n}, o_{n-1}, \ldots, o_{1}, k_{1}\right)
$$

where $o_{i}=\operatorname{ord}_{a} I_{i}$ and $k_{i}=\operatorname{ord}_{a} K_{i}$. Again, this is not too complicated. The motivation for doing so was given in the last chapter. The point is that the components $o_{i}$ and $k_{i}$ do not depend on our choice of the flag $W_{n} \supset \ldots \supset W_{1}$ and of the ideals $J_{i}, I_{i}$ and $K_{i}$ (which, of course, are subject to certain conditions). So it is justified to call $i_{a}(\mathcal{M})$ an invariant of the mobile $\mathcal{M}$ at $a$.

We cheat here a little bit, because in reality, $i_{a}(\mathcal{M})$ has some more components, the combinatorial components $m_{i}$ which are squeezed in between $k_{i}$ and $o_{i-1}$. But all of them are zero except one, and this non-zero component is only used in a very special case in which the mobile is already almost resolved (the so called monomial or combinatorial case, see below and [55]). We do not wish to discuss it in these notes.

We now describe the rules which relate all the local ideals between each other and with the mobile. The problem here is that everything is motivated only a posteriori when you see how the rules make the induction argument work. So we ask you a little patience.

The relation between $J_{i}$ and $I_{i}$ is simple, and prescribed by the $i$-th component $D_{i}$ of the combinatorial handicap. We have $J_{i}=M_{i} \cdot I_{i}$ where $M_{i}=I_{W_{i}}\left(D_{i} \cap W_{i}\right)$ denotes the ideal defining $D_{i} \cap W_{i}$ in $W_{i}$. By the law of transformation for $D_{i}$ and the restrictions on the choice of $W_{i}$, both will intersect transversally so that the factor $I_{W_{i}}\left(D_{i} \cap W_{i}\right)$ is indeed a princi- 
pal monomial ideal. It is the exceptional portion we wish to factor from $J_{i}$, and $I_{i}$ is the interesting part of $J_{i}$ which is not yet resolved. Observe here that $J_{n}$ is just the stalk of $\mathcal{J}$ at $a$, and that at the beginning when all $D_{i}$ are still empty the factorizations trivially exist. After some blowups, it will have to be proven that the factorizations exist, but this will follow directly from the definition of the $D_{i}$. Actually, the transformation law for $D_{i}$ is precisely chosen so as to allow the factorization of $J_{i}$ and moreover so that the factor $I_{i}$ is the weak transform of the respective factor before blowup. It also shows that the component $o_{i}=\operatorname{ord}_{a} I_{i}$ captures interesting information, namely how far $J_{i}$ is from being a principal monomial ideal. The ideal $K_{i}$ equals, up to a small technical detail which we omit, the product of $I_{i}$ with the transversality ideal $Q_{i}=I_{W_{i}}\left(E_{i} \cap W_{i}\right)$ of the mobile $\mathcal{M}$ in dimension $i$. Its order $k_{i}$ (or, equivalently, the difference $q_{i}=k_{i}-o_{i}$ ) measures how far $E_{i}$ and $W_{i}$ are from being separated at $a$. In any case, and this is the important thing, the local top locus of $Q_{i}$ at $a$ will be contained in all components of $E_{i}$ which pass through $a$. This ensures that also the center of blowup will be contained in these components. Recall here that $E_{i}$ collects the dangerous components, i.e., those to which otherwise the chosen virtual center may fail to be transversal.

To repeat: $o_{i}$ tells us how far we are with the resolution of $J_{i}$, and $k_{i}$ how far we are with our transversality problem.

We are left to indicate how we choose the local flag $W_{n} \supset \ldots \supset W_{1}$ and how the ideals in different dimensions relate. As for the flag, $W_{i}$ is a local hypersurface of $W_{i+1}$ at $a$ which is chosen so as to maximize the order at $a$ of the coefficient ideal coeff $W_{i} K_{i+1}$ of $K_{i+1}$ in $W_{i}$. There are several ways how to construct such hypersurfaces, and in characteristic 0 these constructions are particularly nice and behave well. But what is clear and crucial is that the order of coeff $W_{i} K_{i+1}$ does not depend on the choice of $W_{i}$. We then impose our last correlation rule among the various local ideals. It is $J_{i}=\operatorname{coeff}_{W_{i}} K_{i+1}$. Again, there is a slight technical complication which we only sketch. It occurs when $I_{i}$ is already bold regular, i.e., generated by a power of one variable. In this case, the coefficient ideal would be 0 , which is unpleasant for notational regards. Therefore one then sets $J_{i}=1$.

With these settings, it can be shown that the resulting invariant $i_{a}(\mathcal{M})$ is well defined, upper semicontinuous and has all the properties required. In particular, its top locus $Z$ is regular and transversal to the exceptional locus. So $Z$ can be chosen as the center of the next blowup.

It is time that you perform the construction of the local invariant in a concrete example. Only then you will get a feeling for it. Take a principal ideal 
$\mathcal{J}$ in three variables where you are still able to compute the coefficient ideal by hand. You start with trivial handicaps. You get an invariant, and you let $Z$ be its top locus. Then blow up the ambient three-space in this center and consider the transformed mobile above, with new local invariants.

$\mathrm{Ah}$, we have not defined the transform of mobiles yet. Right! Here is the transformation law. Let $a^{\prime}$ be a point of $W^{\prime}$ above $a \in Z$. We only define the transformed mobile $\mathcal{M}^{\prime}$ locally at $a^{\prime}$, and leave it as exercise to show that this also makes sense globally. And we assume that the center $Z$ is the top locus of $i_{a}(\mathcal{M})$ in $W$. This ensures that $Z$ lies in all top loci of the ideals $I_{i}$ and $K_{i}$.

We already said that $\mathcal{J}$ passes to its controlled transform $\mathcal{J}^{\prime}=\mathcal{J}^{\text {! }}$. The control $c^{\prime}$ remains the same $c^{\prime}=c$, except if the order of $\mathcal{J}^{\prime}$ has dropped everywhere below $c$, in which case we are done. The formulas for the combinatorial and transversal handicaps depend on the behaviour of the invariant under blowup. The definition is recursive and a bit involved. So please sharpen your pencil.

We set $D_{n}^{\prime}=D_{n}^{*}+\left(o_{n}-c\right) \cdot Y^{\prime}$ so that $J_{n}^{\prime}=I_{W_{n}^{\prime}}\left(D_{n}^{\prime} \cap W_{n}^{\prime}\right) \cdot I_{n}^{\prime}$ with $I_{n}^{\prime}=I_{n}^{\curlyvee}$ the weak transform of $I_{n}$. We thus dispose of $o_{n}^{\prime}=\operatorname{ord}_{a^{\prime}} I_{n}^{\prime}$. If $o_{n}^{\prime}<o_{n}$ we set $E_{n}^{\prime}=\emptyset$, if $o_{n}^{\prime}=o_{n}$ we set $E_{n}^{\prime}=E_{n}^{*}$ (pullback). Now assume that we have already defined $D_{n}^{\prime}, E_{n}^{\prime}, \ldots, D_{i+1}^{\prime}, E_{i+1}^{\prime}$ in $W^{\prime}$. We thus dispose of the truncated invariant

$$
\left(o_{n}^{\prime}, k_{n}^{\prime}, \ldots, o_{i+1}^{\prime}, k_{i+1}^{\prime}\right)
$$

at $a^{\prime}$. If $\left(o_{n}^{\prime}, k_{n}^{\prime}, \ldots, o_{i+1}^{\prime}, k_{i+1}^{\prime}\right)<_{\text {lex }}\left(o_{n}, k_{n}, \ldots, o_{i+1}, k_{i+1}\right)$ we set $D_{i}^{\prime}=\emptyset$, if $\left(o_{n}^{\prime}, k_{n}^{\prime}, \ldots, o_{i+1}^{\prime}, k_{i+1}^{\prime}\right)=\left(o_{n}, k_{n}, \ldots, o_{i+1}, k_{i+1}\right)$ we set $D_{i}^{\prime}=$ $D_{i}^{*}+\left(o_{i}-k_{i+1}\right) \cdot Y^{\prime}$. We have thus defined also the component $o_{i}^{\prime}$ of our invariant. If $\left(o_{n}^{\prime}, k_{n}^{\prime}, \ldots, k_{i+1}^{\prime}, o_{i}^{\prime}\right)<_{l e x}\left(o_{n}, k_{n}, \ldots, k_{i+1}, o_{i}\right)$ we set $E_{i}^{\prime}=\emptyset$, if $\left(o_{n}^{\prime}, k_{n}^{\prime}, \ldots, k_{i+1}^{\prime}, o_{i}^{\prime}\right)=\left(o_{n}, k_{n}, \ldots, k_{i+1}, o_{i}\right)$ we set $E_{i}^{\prime}=E_{i}^{*}$. So the definition of $D_{i}^{\prime}$ and $E_{i}^{\prime}$ depends on whether the earlier components of the invariant have dropped or not.

The transformation formulas look complicated, but they are precisely chosen so that the ideals $J_{i}^{\prime}, I_{i}^{\prime}, Q_{i}^{\prime}$ and $K_{i}^{\prime}$ satisfy the same rules as their sisters below. This is a computation in local coordinates which is not too difficult. Moreover, whenever $\left(o_{n}^{\prime}, k_{n}^{\prime}, \ldots, o_{i+1}^{\prime}, k_{i+1}^{\prime}\right)$ has not dropped lexicographically, the ideal $I_{i}^{\prime}$ is the weak transform of $I_{i}$ and hence $o_{i}^{\prime} \leq o_{i}$. Similarly, whenever $\left(o_{n}^{\prime}, k_{n}^{\prime}, \ldots, k_{i+1}^{\prime}, o_{i}^{\prime}\right)$ has not dropped lexicographically, the ideal $K_{i}^{\prime}$ is the weak transform of $K_{i}$ and hence $k_{i}^{\prime} \leq k_{i}$. This shows that the invariant never increases. 
To show that it actually decreases, we have to distinguish two circumstances. We place ourselves at the point $a$. Let $d$ be the smallest index so that $o_{d}>0$. We have seen earlier that the center $Z$ then equals $W_{d-1}$. In case that the ideal $K_{d}$ is bold regular (i.e., a power of a variable) and the truncated invariant $\left(o_{n}^{\prime}, k_{n}^{\prime}, \ldots, k_{d+1}^{\prime}, o_{d}^{\prime}\right)$ has not dropped, the transform $K_{d}^{\prime}$ equals the weak transform $K_{d}^{\gamma}=1$ and hence $k_{d}^{\prime}=0<k_{d}$. Note here that in this case $J_{d-1}=1$ by definition and the further components of the invariant are all zero.

The second case is when $K_{d}$ is not bold regular and hence $J_{d-1} \neq 1$. By the choice of $d$ we have $I_{d-1}=1$, so that $J_{d-1}=I_{W_{d-1}}\left(D_{d-1} \cap W_{d-1}\right)$ is a principal monomial ideal. This is the monomial or combinatorial case, in which the hidden components $m_{i}$ of the invariant come into play. To give you a feeling, just think of the polynomial $z^{4}+x^{a} y^{b}$ with $a+b \geq 4$. You should have no problems in figuring out how to choose the center $Z$ (according to the values of $a$ and $b$ ) so that after finitely many blowups the order has dropped below 4 . And in the general case, with $J_{d-1}$ a principal monomial ideal the choice of the center and the reasoning are quite the same.

It looks strange, but we are finished - modulo some breadcrumbs. We have defined the transform $\mathcal{M}^{\prime}$ in $W^{\prime}$ of our mobile $\mathcal{M}$ and given some hints why the local ideals $J_{i}^{\prime}, I_{i}^{\prime}, Q_{i}^{\prime}$ and $K_{i}^{\prime}$ exist again and satisfy the required relations. Actually, the members $W_{i}^{\prime}$ of the local flag $W_{n}^{\prime}, \ldots, W_{1}^{\prime}$ at $a^{\prime}$ coincide with the strict $=$ weak transform of $W_{i}$ if the truncated invariant $\left(o_{n}^{\prime}, k_{n}^{\prime}, \ldots, k_{d+1}^{\prime}, o_{d+1}^{\prime}\right)$ has not dropped at $a^{\prime}$, the remaining members have to be chosen from scratch (which does not matter since the later components of the invariant are irrelevant).

The author of these lines is well aware that the above indications cannot please a critical reader - there is too much hand waving and too little substance, say proof. But precisely this shortcome may motivate you to look at the complete argument as given in the paper [55], and you will realize that there is not so much to add. The constructions are the same (including one or the other additional detail) and they are so systematic that (all) the proofs are really short. No one takes more than half a page, or at most one page.

So how to conclude these notes? One question is whether there is really a need for the non-expert to understand the proof of resolution of singularities in characteristic zero, aside curiosity. There are two answers: First, Hironaka's proposal for the inductive argument - remember that the above is nothing but a conceptualization of the original proof (with the help of 
the techniques developed by the successors of Hironaka) - is a paradigm of mathematical organisation. While reading these notes you should have observed that the clue to everything is the systematic definition of mobiles and their transforms, the rest are almost routine verifications.

Secondly, the problem of resolution is still wide open in positive characteristic and in the arithmetic case. Either somebody invents a completely new approach for these cases (which should not be discarded) or we succeed to understand the characteristic zero proof so much better that we get an idea how to tackle the other cases. Along the lines of Hironaka's proof, when translated to positive characteristic, funny things tend to happen. The invariant may increase, but only in quite special cases which can be pinned down explicitly. And if it increases, the increase is very small, namely at most one (at least in the relevant examples). So you immediately think that if it increases only by one, maybe in the next blowup it drops by two and we have won again. This is almost the case, but only almost. If you are curious to know what type of phenomena may happen, you may look at the article [66]. See you then! 


\section{References}

1. S. Abhyankar. Three dimensional embedded uniformization in characteristic p. Lectures at Purdue University, Notes by M.F. Huang 1968.

2. S. Abhyankar. Local uniformization on algebraic surfaces over ground fields of characteristic $p \neq 0$. Ann. of Math. (2), 63:491-526, 1956.

3. S. Abhyankar. On the valuations centered in a local domain. Amer. J. Math., 78:321-348, 1956.

4. S. Abhyankar. Ramification theoretic methods in algebraic geometry. Princeton Univ. Press, 1959.

5. S. Abhyankar. Current status of the resolution problem. In Summer Institute on Algebraic Geometry. Proc. Amer. Math. Soc., 1964.

6. S. Abhyankar. Resolution of singularities of arithmetical surfaces. In Arithmetical Algebraic Geometry (Proc. Conf. Purdue Univ., 1963), pages 111152. Harper \& Row, New York, 1965.

7. S. Abhyankar. An algorithm on polynomials in one indeterminate with coefficients in a two dimensional regular local domain. Ann. Mat. Pura Appl. (4), 71:25-59, 1966.

8. S. Abhyankar. Resolution of singularities of embedded algebraic surfaces. Pure and Applied Mathematics, Vol. 24. Academic Press, New York, 1966.

9. S. Abhyankar. Three dimensional embedded uniformization on characteristic p. Lectures at Purdue University, Notes by M.F. Huang, 1968.

10. S. Abhyankar. Resolution of singularities of algebraic surfaces. In Algebraic Geometry (Internat. Colloq., Tata Inst. Fund. Res., Bombay, 1968), pages 1-11. Oxford Univ. Press, London, 1969.

11. S. Abhyankar. Desingularization of plane curves. In Singularities, Part 1 (Arcata, Calif., 1981), volume 40 of Proc. Sympos. Pure Math., pages 1-45. Amer. Math. Soc., Providence, RI, 1983.

12. S. Abhyankar. Good points of a hypersurface. Adv. in Math., 68(2):87-256, 1988.

13. S. Abhyankar. Algebraic geometry for scientists and engineers, volume 35 of Mathematical Surveys and Monographs. American Mathematical Society, Providence, RI, 1990.

14. S. Abhyankar and T. T. Moh. Newton-Puiseux expansion and generalized Tschirnhausen transformation. I, II. J. Reine Angew. Math., 260:47-83; ibid. 261 (1973), 29-54, 1973.

15. D. Abramovich and A. J. de Jong. Smoothness, semistability, and toroidal geometry. J. Algebraic Geom., 6(4):789-801, 1997.

16. J. M. Aroca, H. Hironaka, and J. L. Vicente. The theory of the maximal contact. Instituto "Jorge Juan" de Matemáticas, Consejo Superior de Investigaciones Cientificas, Madrid, 1975. Memorias de Matemática del Instituto "Jorge Juan", No. 29. [Mathematical Memoirs of the "Jorge Juan" Institute, No. 29].

17. J. M. Aroca, H. Hironaka, and J. L. Vicente. Desingularization theorems, volume 30 of Memorias de Matemática del Instituto "Jorge Juan" [Mathematical Memoirs of the Jorge Juan Institute]. Consejo Superior de Investigaciones Científicas, Madrid, 1977. 
18. M. Artin. Lipman's proof of resolution of singularities. In J.H. Silverman G. Cornell, editor, Arithmetic Geometry. Springer, 1986.

19. M. Artin. Lipman's proof of resolution of singularities for surfaces. In Arithmetic geometry (Storrs, Conn., 1984), pages 267-287. Springer, New York, 1986.

20. C. Ban and L. J. McEwan. Canonical resolution of a quasi-ordinary surface singularity. Canad. J. Math., 52(6):1149-1163, 2000.

21. MacEwan L. Ban, C. Canonical resolution of a quasi-ordinary surface singularity. Preprint Ohio State University 1998.

22. B. M. Bennett. On the characteristic functions of a local ring. Ann. of Math. (2), 91:25-87, 1970.

23. P. Berthelot. Altérations de variétés algébriques (d'après A. J. de Jong). Astérisque, (241):Exp. No. 815, 5, 273-311, 1997. Séminaire Bourbaki, Vol. 1995/96.

24. E. Bierstone and P. D. Milman. Semianalytic and subanalytic sets. Inst. Hautes Études Sci. Publ. Math., (67):5-42, 1988.

25. E. Bierstone and P. D. Milman. Uniformization of analytic spaces. J. Amer. Math. Soc., 2(4):801-836, 1989.

26. E. Bierstone and P. D. Milman. A simple constructive proof of canonical resolution of singularities. In Effective methods in algebraic geometry (Castiglioncello, 1990), volume 94 of Progr. Math., pages 11-30. Birkhäuser Boston, Boston, MA, 1991.

27. E. Bierstone and P. D. Milman. Canonical desingularization in characteristic zero by blowing up the maximum strata of a local invariant. Invent. Math., 128(2):207-302, 1997.

28. E. Bierstone and P. D. Milman. Resolution of singularities. In Several complex variables (Berkeley, CA, 1995-1996), volume 37 of Math. Sci. Res. Inst. Publ., pages 43-78. Cambridge Univ. Press, Cambridge, 1999.

29. E. Bierstone and P. D. Milman. Desingularization algorithms. I. Role of exceptional divisors. Mosc. Math. J., 3(3):751-805, 1197, 2003. Dedicated to Vladimir Igorevich Arnold on the occasion of his 65 th birthday.

30. G. Bodnár. Computation of blowing up centers. J. Pure Appl. Algebra, 179(3):221-233, 2003.

31. G. Bodnár and J. Schicho. Automated resolution of singularities for hypersurfaces. J. Symbolic Comput., 30(4):401-428, 2000.

32. G. Bodnár and J. Schicho. A computer program for the resolution of singularities. In Resolution of singularities (Obergurgl, 1997), volume 181 of Progr. Math., pages 231-238. Birkhäuser, Basel, 2000.

33. G. Bodnár and J. Schicho. Two computational techniques for singularity resolution. J. Symbolic Comput., 32(1-2):39-54, 2001. Computer algebra and mechanized reasoning (St. Andrews, 2000).

34. F. A. Bogomolov and T. G. Pantev. Weak Hironaka theorem. Math. Res. Lett., 3(3):299-307, 1996.

35. M. Brandenberg. Aufblasungen affiner Varietäten. PhD thesis, 1992. Thesis Zürich.

36. A. Bravo and O. Villamayor. A strengthening of resolution of singularities 
in characteristic zero. Proc. London Math. Soc. (3), 86(2):327-357, 2003.

37. A. M. Bravo, S. Encinas, and O. Villamayor. A simplified proof of desingularization and applications. Rev. Mat. Iberoamericana, 21(2):349-458, 2005.

38. E. Brieskorn. Über die Auflösung gewisser Singularitäten von holomorphen Abbildungen. Math. Ann., 166:76-102, 1966.

39. E. Brieskorn. Die Auflösung der rationalen Singularitäten holomorpher Abbildungen. Math. Ann., 178:255-270, 1968.

40. E. Brieskorn and H. Knörrer. Ebene algebraische Kurven. Birkhäuser Verlag, Basel, 1981.

41. F. Cano and R. Piedra. Characteristic polygon of surface singularities. In Géométrie algébrique et applications, II (La Rábida, 1984), volume 23 of Travaux en Cours, pages 27-42. Hermann, Paris, 1987.

42. V. Cossart. Desingularization of embedded excellent surfaces. Tôhoku Math. J. (2), 33(1):25-33, 1981.

43. V. Cossart. Polyèdre caractéristique et éclatements combinatoires. Rev. Mat. Iberoamericana, 5(1-2):67-95, 1989.

44. V. Cossart. Contact maximal en caractéristique positive et petite multiplicité. Duke Math. J., 63(1):57-64, 1991.

45. V. Cossart. Uniformisation et désingularisation des surfaces d'après Zariski. In Resolution of singularities (Obergurgl, 1997), volume 181 of Progr. Math., pages 239-258. Birkhäuser, Basel, 2000.

46. V. Cossart, J. Giraud, and U. Orbanz. Resolution of surface singularities, volume 1101 of Lecture Notes in Mathematics. Springer-Verlag, Berlin, 1984. With an appendix by H. Hironaka.

47. S. D. Cutkosky. When are the blow ups of two ideals the same scheme? Comm. Algebra, 19(4):1119-1124, 1991.

48. S. D. Cutkosky. Local factorization of birational maps. Adv. Math., 132(2):167-315, 1997.

49. S. D. Cutkosky. Local monomialization and factorization of morphisms. Astérisque, (260):vi+143, 1999.

50. S. D. Cutkosky. Monomialization of morphisms from 3-folds to surfaces, volume 1786 of Lecture Notes in Mathematics. Springer-Verlag, Berlin, 2002.

51. S. D. Cutkosky. Resolution of morphisms. In Valuation theory and its applications, Vol. I (Saskatoon, SK, 1999), volume 32 of Fields Inst. Commun., pages 117-140. Amer. Math. Soc., Providence, RI, 2002.

52. S. D. Cutkosky. Generically finite morphisms and simultaneous resolution of singularities. In Commutative algebra (Grenoble/Lyon, 2001), volume 331 of Contemp. Math., pages 75-99. Amer. Math. Soc., Providence, RI, 2003.

53. S. D. Cutkosky. Resolution of singularities, volume 63 of Graduate Studies in Mathematics. American Mathematical Society, Providence, RI, 2004.

54. D. Eisenbud and J. Harris. The geometry of schemes, volume 197 of Graduate Texts in Mathematics. Springer-Verlag, New York, 2000.

55. S. Encinas and H. Hauser. Strong resolution of singularities in characteristic zero. Comment. Math. Helv., 77(4):821-845, 2002.

56. S. Encinas and O. Villamayor. Good points and constructive resolution of singularities. Acta Math., 181(1):109-158, 1998. 
57. S. Encinas and O. Villamayor. A course on constructive desingularization and equivariance. In Resolution of singularities (Obergurgl, 1997), volume 181 of Progr. Math., pages 147-227. Birkhäuser, Basel, 2000.

58. S. Encinas and O. Villamayor. A new proof of desingularization over fields of characteristic zero. In Proceedings of the International Conference on Algebraic Geometry and Singularities (Spanish) (Sevilla, 2001), volume 19, pages 339-353, 2003.

59. J. Giraud. Desingularization in low dimension, volume Resolution of surface singularities of Lecture Notes in Math. Springer 1984, 51-78.

60. J. Giraud. Étude locale des singularités. U.E.R. Mathématique, Université Paris XI, Orsay, 1972. Cours de 3ème cycle, 1971-1972, Publications Mathématiques d'Orsay, No. 26.

61. J. Giraud. Sur la théorie du contact maximal. Math. Z., 137:285-310, 1974.

62. J. Giraud. Contact maximal en caractéristique positive. Ann. Sci. École Norm. Sup. (4), 8(2):201-234, 1975.

63. R. Goldin and B. Teissier. Resolving singularities of plane analytic branches with one toric morphism. In Resolution of singularities (Obergurgl, 1997), volume 181 of Progr. Math., pages 315-340. Birkhäuser, Basel, 2000.

64. A. Grothendieck. Travaux de Heisouké Hironaka sur la résolution des singularités. In Actes du Congrès International des Mathématiciens (Nice, 1970), Tome 1, pages 7-9. Gauthier-Villars, Paris, 1971.

65. H. Hauser. Seven short stories on blowups and resolution. Proceedings of the Gökova Geometry Topology Conference 2005, pp. 1-48. International Press 2006.

66. H. Hauser. Why Hironaka's resolution fails in positive characteristic. preprint on www.hh.hauser.cc.

67. H. Hauser. Seventeen obstacles for resolution of singularities. In Singularities (Oberwolfach, 1996), volume 162 of Progr. Math., pages 289-313. Birkhäuser, Basel, 1998.

68. H. Hauser. Excellent surfaces and their taut resolution. In Resolution of singularities (Obergurgl, 1997), volume 181 of Progr. Math., pages 341-373. Birkhäuser, Basel, 2000.

69. H. Hauser. Resolution of singularities 1860-1999. In Resolution of singularities (Obergurgl, 1997), volume 181 of Progr. Math., pages 5-36. Birkhäuser, Basel, 2000.

70. H. Hauser. The Hironaka theorem on resolution of singularities (or: A proof we always wanted to understand). Bull. Amer. Math. Soc. (N.S.), 40(3):323403 (electronic), 2003.

71. H. Hauser. Three power series techniques. Proc. London Math. Soc. (3), 89(1):1-24, 2004.

72. H. Hauser, J. Lipman, F. Oort, and A. Quirós, editors. Resolution of singularities, volume 181 of Progress in Mathematics. Birkhäuser Verlag, Basel, 2000. A research textbook in tribute to Oscar Zariski, Papers from the Working Week held in Obergurgl, September 7-14, 1997.

73. H. Hauser and G. Regensburger. Explizite Auflösung von ebenen Kurvensingularitäten in beliebiger Charakteristik. Enseign. Math. (2), 50(3-4):305- 
353, 2004.

74. M. Herrmann, S. Ikeda, and U. Orbanz. Equimultiplicity and blowing up. Springer-Verlag, Berlin, 1988. An algebraic study, With an appendix by B. Moonen.

75. H. Hironaka. Desingularization of excellent surfaces. Notes by B. Bennett at the Conference on Algebraic Geometry, Bowdoin 1967. Reprinted in: Cossart, V., Giraud, J., Orbanz, U.: Resolution of surface singularities. Lecture Notes in Mathematics 1101, Springer 1984.

76. H. Hironaka. Preface to Zariski's collected papers i, 307-312, mit press 1979.

77. H. Hironaka. Resolution of singularities of an algebraic variety over a field of characteristic zero. I, II. Ann. of Math. (2) 79 (1964), 109-203; ibid. (2), 79:205-326, 1964.

78. H. Hironaka. Characteristic polyhedra of singularities. J. Math. Kyoto Univ., 7:251-293, 1967.

79. H. Hironaka. Additive groups associated with points of a projective space. Ann. of Math. (2), 92:327-334, 1970.

80. H. Hironaka. Certain numerical characters of singularities. J. Math. Kyoto Univ., 10:151-187, 1970.

81. H. Hironaka. Desingularization of complex-analytic varieties. In Actes $d u$ Congrès International des Mathématiciens (Nice, 1970), Tome 2, pages 627631. Gauthier-Villars, Paris, 1971.

82. H. Hironaka. Schemes, etc. In Algebraic geometry, Oslo 1970 (Proc. Fifth Nordic Summer School in Math.), pages 291-313. Wolters-Noordhoff, Groningen, 1972.

83. H. Hironaka. Bimeromorphic smoothing of a complex-analytic space. Acta Math. Vietnam., 2(2):103-168, 1977.

84. H. Hironaka. Idealistic exponents of singularity. In Algebraic geometry (J. J. Sylvester Sympos., Johns Hopkins Univ., Baltimore, Md., 1976), pages 52-125. Johns Hopkins Univ. Press, Baltimore, Md., 1977.

85. H. Hironaka. Stratification and flatness. In Real and complex singularities (Proc. Ninth Nordic Summer School/NAVF Sympos. Math., Oslo, 1976), pages 199-265. Sijthoff and Noordhoff, Alphen aan den Rijn, 1977.

86. H. Hironaka. On the presentations of resolution data, volume Algebraic Analysis, Geometry and Number Theory 1988. 1989.

87. H. Jung. Darstellung der Funktionen eines algebraischen Körpers zweier unabhängiger Veränderlicher $\mathrm{x}, \mathrm{y}$ in der Umgebung einer Stelle $\mathrm{x}=\mathrm{a}, \mathrm{y}=\mathrm{b}$. J. Reine Angew. Math., 133:289-314, 1908.

88. K. Kiyek and J. L. Vicente. Resolution of curve and surface singularities, volume 4 of Algebras and Applications. Kluwer Academic Publishers, Dordrecht, 2004.

89. J. Kollár. Lecutres on resolution of singularities. Preprint Princeton 2005.

90. E. Kunz. Algebraische Geometrie IV. Vorlesung Regensburg.

91. D. T. Le and M. Oka. On resolution complexity of plane curves. Kodai Math. J., 18:1-36, 1995.

92. M. Lejeune and B. Teissier. Contribution à l'étude des singularités du point de vue du polygone de Newton. Thèse d'Etat, Paris 1973. 
93. J. Lipman. Rational singularities, with applications to algebraic surfaces and unique factorization. Inst. Hautes Études Sci. Publ. Math., (36):195279, 1969.

94. J. Lipman. Introduction to resolution of singularities. In Algebraic geometry (Proc. Sympos. Pure Math., Vol. 29, Humboldt State Univ., Arcata, Calif., 1974), pages 187-230. Amer. Math. Soc., Providence, R.I., 1975.

95. J. Lipman. Desingularization of two-dimensional schemes. Ann. Math. (2), 107(1):151-207, 1978.

96. J. Lipman. On complete ideals in regular local rings. In Algebraic geometry and commutative algebra, Vol. I, pages 203-231. Kinokuniya, Tokyo, 1988.

97. J. Lipman. Topological invariants of quasi-ordinary singularities. Mem. Amer. Math. Soc., 74(388):1-107, 1988.

98. T. T. Moh. On a stability theorem for local uniformization in characteristic p. Publ. Res. Inst. Math. Sci., 23(6):965-973, 1987.

99. T. T. Moh. Quasi-canonical uniformization of hypersurface singularities of characteristic zero. Comm. Algebra, 20(11):3207-3249, 1992.

100. T. T. Moh. On a Newton polygon approach to the uniformization of singularities of characteristic $p$. In Algebraic geometry and singularities (La Rábida, 1991), volume 134 of Progr. Math., pages 49-93. Birkhäuser, Basel, 1996.

101. H. T. Muhly and O. Zariski. The Resolution of Singularities of an Algebraic Curve. Amer. J. Math., 61(1):107-114, 1939.

102. S. B. Mulay. Equimultiplicity and hyperplanarity. Proc. Amer. Math. Soc., 89(3):407-413, 1983.

103. R. Narasimhan. Hyperplanarity of the equimultiple locus. Proc. Amer. Math. Soc., 87(3):403-408, 1983.

104. R. Narasimhan. Monomial equimultiple curves in positive characteristic. Proc. Amer. Math. Soc., 89(3):402-406, 1983.

105. T. Oda. Hironaka's additive group scheme. In Number theory, algebraic geometry and commutative algebra, in honor of Yasuo Akizuki, pages 181-219. Kinokuniya, Tokyo, 1973.

106. T. Oda. Hironaka group schemes and resolution of singularities. In Algebraic geometry (Tokyo/Kyoto, 1982), volume 1016 of Lecture Notes in Math., pages 295-312. Springer, Berlin, 1983.

107. T. Oda. Hironaka's additive group scheme. II. Publ. Res. Inst. Math. Sci., 19(3):1163-1179, 1983.

108. T. Oda. Infinitely very near singular points. In Complex analytic singularities, volume 8 of Adv. Stud. Pure Math., pages 363-404. North-Holland, Amsterdam, 1987.

109. M. Oka. Geometry of plane curves via toroidal resolution. In Algebraic geometry and singularities (La Rábida, 1991), volume 134 of Progr. Math., pages 95-121. Birkhäuser, Basel, 1996.

110. U. Orbanz. Embedded resolution of algebraic surfaces after Abhyankar (characteristic 0), volume Resolution of surface singularities of Lecture Notes in Math. Springer 1984.

111. J. Pfeifle. Aufblasung monomialer Ideale. Master's thesis, Diplomarbeit 
Innsbruck, 1996.

112. C. Rodriguez Sanchez. Good points and local resolution of threefold singularities. PhD thesis, Thesis, Univ. Leon, 1998.

113. J. Rosenberg. Blowing up nonreduced toric subschemes of $\mathbb{A}^{n}$. Preprint 1998.

114. B. Singh. Effect of a permissible blowing-up on the local Hilbert functions. Invent. Math., 26:201-212, 1974.

115. B. Singh. Formal invariance of local characteristic functions. In Seminar D. Eisenbud/B. Singh/W. Vogel, Vol. 1, volume 29 of Teubner-Texte zur Math., pages 44-59. Teubner, Leipzig, 1980.

116. M. Spivakovsky. A counterexample to Hironaka's "hard" polyhedra game. Publ. Res. Inst. Math. Sci., 18(3):1009-1012, 1982.

117. M. Spivakovsky. A solution to Hironaka's polyhedra game. In Arithmetic and geometry, Vol. II, volume 36 of Progr. Math., pages 419-432. Birkhäuser Boston, Boston, MA, 1983.

118. M. Spivakovsky. A counterexample to the theorem of Beppo Levi in three dimensions. Invent. Math., 96(1):181-183, 1989.

119. B. Teissier. Valuations, deformations, and toric geometry. In Valuation theory and its applications, Vol. II (Saskatoon, SK, 1999), volume 33 of Fields Inst. Commun., pages 361-459. Amer. Math. Soc., Providence, RI, 2003.

120. W. V. Vasconcelos. Arithmetic of blowup algebras, volume 195 of London Mathematical Society Lecture Note Series. Cambridge University Press, Cambridge, 1994.

121. O. Villamayor. Constructiveness of Hironaka's resolution. Ann. Sci. École Norm. Sup. (4), 22(1):1-32, 1989.

122. O. Villamayor. Patching local uniformizations. Ann. Scient. Ec. Norm. Sup. Paris, 25:629-677, 1992.

123. O. Villamayor. An introduction to the algorithm of resolution. In L. Narváez A. Campillo, editor, Algebraic Geometry and Singularities. Birkhaeuser, 1996. Proc. Conf. on Singularities La Rábida.

124. O. Villamayor. On equiresolution and a question of Zariski. Acta Math., 185:123-159, 2000.

125. R. J. Walker. Reduction of the singularities of an algebraic surface. Ann. of Math. (2), 36(2):336-365, 1935.

126. R. J. Walker. Algebraic curves. Dover Publications Inc., New York, 1962.

127. B. Youssin. Newton polyhedra without coordinates. Mem. Amer. Math. Soc., 87(433):i-vi, 1-74, 1990.

128. O. Zariski. Polynomial Ideals Defined by Infinitely Near Base Points. Amer. J. Math., 60(1):151-204, 1938.

129. O. Zariski. The reduction of the singularities of an algebraic surface. Ann. of Math. (2), 40:639-689, 1939.

130. O. Zariski. Local uniformization on algebraic varieties. Ann. of Math. (2), 41:852-896, 1940.

131. O. Zariski. Normal varieties and birational correspondences. Bull. Amer. Math. Soc., 48:402-413, 1942.

132. O. Zariski. A simplified proof for the resolution of singularities of an alge- 
braic surface. Ann. of Math. (2), 43:583-593, 1942.

133. O. Zariski. The compactness of the Riemann manifold of an abstract field of algebraic functions. Bull. Amer. Math. Soc., 50:683-691, 1944.

134. O. Zariski. Reduction of the singularities of algebraic three dimensional varieties. Ann. of Math. (2), 45:472-542, 1944.

135. O. Zariski. The concept of a simple point of an abstract algebraic variety. Trans. Amer. Math. Soc., 62:1-52, 1947.

136. O. Zariski. Exceptional singularities of an algebroid surface and their reduction. Atti Accad. Naz. Lincei Rend. Cl. Sci. Fis. Mat. Natur. (8), 43:135146, 1967.

137. O. Zariski. Collected papers. Vol. I: Foundations of algebraic geometry and resolution of singularities. The MIT Press, Cambridge, Mass.-London, 1972. Edited by H. Hironaka and D. Mumford, Mathematicians of Our Time, Vol. 2 .

138. O. Zariski. A new proof of the total embedded resolution theorem for algebraic surfaces (based on the theory of quasi-ordinary singularities). Amer. J. Math., 100(2):411-442, 1978.

139. O. Zariski. Collected papers. Vol. IV, volume 16 of Mathematicians of Our Time. MIT Press, Cambridge, Mass., 1979. Equisingularity on algebraic varieties, Edited and with an introduction by J. Lipman and B. Teissier.

140. O. Zariski. Algebraic surfaces. Classics in Mathematics. Springer-Verlag, Berlin, 1995. With appendices by S. S. Abhyankar, J. Lipman and D. Mumford, Preface to the appendices by Mumford, Reprint of the second (1971) edition. 